\title{
Structural Optimization of downhole oil-water separator
}

2 Abstract: Downhole oil-water separator is the most important component of downhole oil-water separation system.

3 Its structure has great effects on oil concentration of underflow (OCU) thus requiring further optimization. This work aims to propose an optimization method for the structural optimization of downhole oil-water separator that consists of two series de-oiling hydrocyclones. Firstly, the significant factors influencing OCU are identified by two-level Plackett-Burman Design (PBD) with twelve factors. Then the central levels of the five significant factors identified from PBD are determined by steepest ascent design. Secondly, response surface methodology (RSM) is used to establish the second order model between the OCU and five significant factors for the implementation of

9 particle swarm optimization (PSO). Finally, the optimal structural parameters are obtained by PSO algorithm.

10 Computational fluid dynamics (CFD) is employed to calculate the OCU for each particular case and analyze the

11 separation performance variations before and after optimization. The simulation results show that compared with

12 the original geometry and the best geometry in CCF design, the OCU of the optimized decreases. And the 13 separation performance of the optimized geometry is remarkably improved for the oil droplets whose particle sizes 14 are smaller than $35.78 \mu \mathrm{m}$. Furthermore, laboratory experiments have been conducted to validate the proposed 15 optimization method. The experimental results confirm that the OCU also reduces following the proposed 16 optimization method. It can be summarized that PSO algorithm combined with PBD, steepest ascent design and 17 RSM can be an effective method for the structural optimization of the downhole oil-water separator.

18 Keywords: Downhole oil-water separator Response surface methodology Particle swarm optimization

19 Numerical simulation Structural Optimization

\section{1. Introduction}

21 Downhole oil-water separation is an efficient, environmental-friendly and energy-saving technology, which 22 can realize a complete production process including oil-water recovery, separation, oil lift and water injection in the 23 same wellbore (Ogunsina and Wiggins, 2005; Amini et al., 2012). Downhole oil-water separator is the most 24 important device of downhole oil-water separation system, and can determine the oil-water separation effect. In 25 order to preventing the injection layer (waste layer or formations with high permeability) from being blocked, the 26 oil concentration of the liquid injected to the injection layer should be as small as possible (Scaramuzza et al., 27 2001). In other words, the higher the separation efficiency of downhole oil-water separator, the smaller the OCU. 28 To improve the separation performance, the scheme of two de-oiling hydrocyclones in series is often adopted in the 
29 design of downhole oil-water separator. Typically, a de-oiling hydrocyclone (DOHC) is composed of one inlet 30 stream (tangential inlet in the cylindrical section) and two outlet streams, that is overflow stream with higher oil 31 concentration and underflow stream with higher water concentration (Amini et al., 2012). Compared with the 32 solid-liquid hydrocyclone (SLHC), it is more difficult to improve the separation efficiency of DOHC due to low 33 density differences between two liquids in the DOHC. The DOHC generally follow a conventional design and may 34 have some limitations on separation performance (Ghodrat et al., 2014a). Therefore, many previous works have 35 been done to optimize the geometry of DOHC.

36 Early, experiments and theoretical analysis are the main method to study hydrocyclones. In the1960s, the 37 hydrocyclone used for oil-water separation was firstly studied by a group of researchers from Southampton 38 University (Thew, 1986). They designed a typical DOHC with two cone sections and obtained some optimal 39 parameters. In particular, the ratio of the inlet diameter to the reference diameter was 0.35 ; the ratio of the tailpipe 40 diameter to the reference diameter was 20 . They also found that the large cone section with an angle of $20^{\circ}$ and the 41 small cone section with an angle of $1.5^{\circ}$ could enhance separation efficiency. Young et al. (1994) proposed a modified design called Amoco DOHC with a single cone section. They found that the optimal ratio of the inlet 43 diameter to the reference diameter was 0.25 , and the optimal cone angle was $6^{\circ}$. And a very slightly cone tailpipe 44 was better than a cylindrical tailpipe. For the droplet whose particle size smaller than $20 \mu \mathrm{m}$, the Amoco DOHC could raise separation efficiency by $20 \%$ compared with the DOHC with two cone sections. Thew (2000) proposed the rectangular tangential inlet introduced through a gentle volute. Li et al. (2011a) found that the average separation efficiency of the DOHC using rectangular tangential inlet could be up to $99.4 \%$ in their experimental studies. Li et al. (2011b) also conducted experiments and found that the DOHC with cylinder-cone-inverted cone overflow pipe reduced the short-circuit flow, and the average separation efficiency of the DOHC could be up to 98.1\%. Belaidi and Thew (2003) proposed a new modified design to increase the separation of free gas based on the typical DOHC. In this design, a tapered swirl chamber was used to separate gas at entry, a twin-tangential inlet was employed to provide a stable and axisymmetric flow and a total angle of $2^{\circ}$ was adopted to accelerate the swirling stream.

In recent years, CFD has been widely used in the analysis of internal flow field and structural optimization of DOHC (Ghodrat et al., 2014b). Noroozi et al. (2009) investigated the effects of four different inlet designs on the DOHC efficiency using an Eulerian-Eulerian three-dimensional CFD model. Their simulations showed that the 
57 separation efficiency could be improved approximately $10 \%$ when using a helical form of inlet. Noroozi and

58 Hashemabadi (2011) studied the effects of different inlet chamber designs (exponential, conical, quadratic 59 polynomial body profile and standard design) on efficiency. The simulation results indicated that the separation 60 efficiency could be improved about $8 \%$ using exponential body shape. Saidi et al. (2013) investigated the effects of 61 different cone angles $\left(6^{\circ}, 10^{\circ}\right.$ and $\left.20^{\circ}\right)$ on the flow field and separation efficiency of the DOHC using large eddy 62 simulation. As a result, the design with $6^{\circ}$ cone angle showed the best separation performance. However, no specific optimization techniques are used for systematic optimization of DOHC in the above 64 works. Chu et al. (2000) investigated the influence of body and inlet chamber design of SLHC on separation 65 efficiency using orthogonal design method. Vieira et al. (2011) studying the effect of geometric parameters on the performance of SLHC by CFD and RSM, found a better design leading to Euler number and cut size in minimum levels. Silva et al. (2012) used the differential evolution algorithm to improve the performance of SLHC. The optimized configuration of hydrocyclone presented a high efficiency and a small reduced cut size. Silva et al, (2015) obtained an optimized geometry for a filtering hydrocyclone with maximum total efficiency by the differential evolution algorithm. However, these optimizations were proposed for single SLHC, and no work has been done to optimize the geometry of downhole oil-water separator that consists of two series DOHCs. Compared with single DOHC or SLHC, there are too many structural parameters to be optimized for the downhole oil-water separator including two series DOHCs. A large number of experiments need to be conducted by using full factorial experiment. Thus, it is very necessary to find a suitable optimization method, which can not only save time and costs, but also can find the optimal solution efficiently. Zhao et al. (2014b) adopted orthogonal experimental design to optimize nine structural parameters of a downhole dual-stage tandem hydrocyclone. The results showed that after optimization, an effective reduction of OCU was attained by this method. However, in the orthogonal experimental design, the levels of factors were only several discrete points, the structural parameters obtained may not the optimal values.

80 Previous laboratory experiments showed that under the rated flow rate $\left(30 \mathrm{~m}^{3} / \mathrm{d}\right)$, the OCU of the unoptimized 81 downhole oil-water separator was not low enough for downhole application (Zhao et al., 2013; Zhao et al., 2014a).

82 For the downhole oil-water separator, the OCU or the separation efficiency is the most important index for 83 performance evaluation. Considering the geometry design of hydrocyclone has great effects on OCU (Chu et al., 84 2000), further optimization of downhole oil-water separator is required. This investigation tries to optimize the 
85 geometry of downhole oil-water separator using PSO algorithm combined with PBD, steepest ascent design and

86 RSM. After optimization, the variations of structural parameters and separation performance are investigated.

87 Finally, laboratory experiments with the geometry obtained from the optimization study are conducted to validate 88 the proposed optimization method.

\section{2. Structure and methods}

90 2.1. Structure of downhole oil-water separator

91 As shown in the dashed box of Fig. 1a, the downhole oil-water separator is suitable for low productivity well 92 using screw pump to extract oil (Zhao and Li, 2011; Zhao et al., 2014a). It mainly includes upper cross-channel 93 nipple, the first DOHC, central cross-channel nipple, the second DOHC, under cross-channel nipple and shell. Each 94 DOHC consists of cylindrical section, large cone section, small cone section and tail pipe section as shown in Fig.

95 1b. The arc tangential inlet with gradual rectangular cross section is specially designed for the two DOHCs, as 96 shown in Fig. 2. Table 1 shows the typical structural parameters of the two DOHCs attained from previous studies 97 (Zhao et al., 2014a).

The working principle is as follows. Firstly, the oil-water mixture from reservoirs passes through the internal 99 channels in the downhole oil-water separator, including the annular between the inner pipe and outer pipe and the 100 flow channel $\mathrm{k}, \mathrm{j}, \mathrm{e}, \mathrm{c}$ and $\mathrm{b}$, entering into the screw pump, which is just located above the downhole oil-water 101 separator. Then the liquid pressurized by the screw pump enters into the first DOHC through the flow channel d, $\mathrm{f}$ and g. It should be noted that at the beginning, a part of the pressurized liquid may go up to the wellhead along the 103 tubing. But it still maintains high pressure level due to the proper sealing at the wellhead. After the first separation, 104 the oil-rich liquid passes through the first overflow channel a (oriented perpendicular to the paper) into the 105 tubing-casing annular and is lifted to the ground. And the water-rich liquid passes through channel g into the second 106 DOHC. After the second separation, the newly oil-rich liquid passes through the first overflow channel i (oriented 107 perpendicular to the paper) into the tubing-casing annular and is also lifted to the ground. Finally, the liquid with trace oil goes into the inner pipe and is injected into the injection layer.

Based on the results obtained by our research group and other researchers (Thew, 1986; Young et al., 1994;

110 Thew, 2000; Chu et al., 2000; Delfos et al., 2004; Kharoua et al., 2010; Li et al., 2011b; Noroozi and Hashemabadi, 111 2011; Ghodrat et al., 2014a; Saidi et al., 2013; Ghodrat et al., 2014b; Silva et al., 2015), the structural parameters 112 that have effects on OCU can be identified: overflow diameter $D_{\mathrm{o} 1}$ and $D_{\mathrm{o} 2}$, cylindrical length $L_{\mathrm{c} 1}$ and $L_{\mathrm{c} 2}$, large 
113 cone angle $\alpha_{1}$ and $\alpha_{2}$, small cone angle $\beta_{1}$ and $\beta_{2}$, tail pipe diameter $D_{\mathrm{u} 1}$ and $D_{\mathrm{u} 2}$, tail pipe length $L_{\mathrm{u} 1}$ and $L_{\mathrm{u} 2}$. The

114 inlet shape and its parameters maintain unchanged.

115 2.2. Design of experiments and statistical analysis

\section{2.2.1. Plackett-Burman design}

117 Even though there are twelve factors (structural parameters) influencing OCU to be optimized, not all of them

118 are critical factors. PBD is an efficient screening method to identify the significant factors among lots of factors

119 that affects a process (Priyadharshini and Bakthavatsalam, 2016). In order to reduce unnecessary experiments and

120 get satisfactory results, PBD is used to select the significant factors out of the twelve factors considered in this 121 study.

$122 \quad$ 2.2.2 Steepest ascent design

123 Only in the small neighborhood of the investigated area, the predicted value attained by regression equation 124 can be fully close to the true value. In other areas, the difference between the true value and predicted value is 125 usually very large. In order to establish the effective regression equation using RSM, the central level of factors 126 should approach the optimal value. Steepest ascent design uses the variable gradient of the experimental value as 127 the ascent path, which can be rapidly and economically approach the optimal value (Dong et al., 2003; Yao et al., 128 2013). Thus, steepest ascent design is selected to determine the central level of the five significant factors obtained 129 by PBD.

\section{$130 \quad$ 2.2.3 Response surface methodology}

131 To attain the optimal solution using PSO algorithm, the mathematical relationship of the OCU to the five 132 significant factors must be determined. RSM is a statistical method of multifactorial analysis of experimental data 133 which provides a better understanding of the process than the standard experimental methods, because it is able to 134 predict how the inputs influence the outputs in a complex process where different factors can interact among 135 themselves (Šereš et al., 2010). Central composite face-centered (CCF) design is frequently used in RSM because 136 of its advantages in optimizing multifactor problems with optimum number of experimental runs (Mangili et al., 137 2015). Therefore, the RSM based on CCF design is chosen to build the mathematical model between the OCU and 138 five significant factors and analyze the effect of each factor and their interactions.

139 Due to the desirable properties of second order polynomials, such as high predictability, robustness and 140 simplicity, they are often used for estimating response value and determining the size of effects (Karami et al., 
141 2016). The general form of a second order model is as below:

$$
y=\beta_{0}+\sum_{i=1}^{k} \beta_{i} x_{i}+\sum_{i=1}^{k} \beta_{i i} x_{i}^{2}+\sum_{i<j}^{k} \beta_{i j} x_{i} x_{j}+\varepsilon
$$

143 Where $y$ is the purposed response, here, it is OCU; $x_{i}$ values are the $i$ th independent variables of the function; $\varepsilon$

144 value shows random error; $\beta_{0}$ is a real constant of regression; $\beta_{i}, \beta_{j}, \beta_{i i}$ and $\beta_{i j}$ values are regression coefficients of 145 the main, interaction and quadratic terms.

146 2.2.4. Statistical analysis

147 Statistical analysis of the model is performed to evaluate the analysis of variance (ANOVA). Analysis includes $148 \quad F$-test associated with probability $P(F)$ and the coefficient of determination $\left(R^{2}\right)$ which measure the goodness of fit 149 of the regression model. The response surface and contour plots of predicted responses of the model are used to assess the interaction between the significant factors. Minitab 17 is used for designing experiments as well as for regression analysis of the experimental data obtained.

2.3 Particle swarm optimization algorithm

In this study, PSO algorithm is employed to search the optimal solution of the equation (the mathematical relationship of the OCU to five significant factors) given by RSM. PSO algorithm is a stochastic population-based optimization technique for solving global optimization problems developed by Eberhart and Kennedy in 1995 (Dong et al., 2005). It is an evolutionary computation technique that starts with a crowd of grain called as the swarm and mainly based on social models, such as bird flocking, fish schooling and the swarm theory (Rao and Patel, 2010). The basic equations of PSO algorithm (Rao and Patel, 2010) are as follows:

$V_{i d}(t+1)=\omega \cdot V_{i d}(t)+c_{1} \cdot \operatorname{rand}() \cdot\left(p_{i d}-x_{i d}(t)\right)+c_{2} \cdot \operatorname{rand}() \cdot\left(p_{g d}-x_{i d}(t)\right)$

$x_{i}(t+1)=x_{i}(t)+V_{i}(t+1)$

161 Where $V_{i d}$ is the velocity of particle $i$ in $d$-dimensional space, $x_{i}(t)=\left(x_{i 1}, x_{i 2}, x_{i 3}, \cdots \cdots x_{i n}\right)$ is a vector with length $n$, and represents the position of particle $i$ in $i$ th generation; $V_{i}(t)=\left(V_{i 1}, V_{i 2}, V_{i 3}, \cdots \cdots V_{i n}\right)$ is also a vector with length $n$, and represents the velocity of particle $i$ in $i$ th generation; $\omega$ represents inertia coefficient; $c_{1}$ and $c_{2}$ are the acceleration constants with positive values, and usually, $c_{1}=c_{2}=2$ (Bergh and Engelbrecht, 2006; Dong et al., 2005; Rao and Patel, 2010); $\operatorname{rand}()$ is a random number in $[0,1] ; p_{\text {id }}$ is the individual extreme value, $p_{g \mathrm{gd}}$ is the global extreme value. 
(1998), which can be expressed as:

170 Where $T_{\max }$ is the maximum iteration number; $\omega_{\mathrm{ini}}$ is the initial inertia weight; $\omega_{\mathrm{end}}$ is the inertia weight when $t=$

$171 T_{\max } ;$ in general, $\omega_{\text {ini }}=0.9, \omega_{\text {end }}=0.4$.

172 2.4. Numerical simulation method

173 Fluent CFD software is chosen to simulate multiphase (oil and water) flow field in downhole oil-water

174 separator and calculate OCU. The oil-water flow behavior and interphase interaction are simulated by using 175 algebraic slip mixture model (Grady et al., 2003; Noroozi and Hashemabadi, 2011). And the complex turbulent 176 behavior is described by the large eddy simulation model (Brennan, 2006).

Gambit 2.4.6 is employed to perform the geometry generation and meshing. According to the original structure of downhole oil-water separator, the three dimensional model of flow domain is built, as shown in Fig. 3 . The three dimensional grid of flow domain is generated by using the method proposed by Zhao et al. (2014a). In order to eliminate the influence of grid density on the numerical result and save calculation time, using the method introduced by Noroozi et al. (2009) analyzes the grid independency and determine the grid number.

The physical parameters of materials under normal temperature and pressure are shown in Table 2. In addition, Table 3 shows the setting of boundary condition in this study. According to the actual working conditions, the boundary conditions are set as follows.

The boundary style of inlet is set to velocity-inlet. According to the rated flow rate and the cross-sectional area of the inlet (Fig. 3), velocity at the inlet can be calculated. The outlets including the first overflow, second overflow and underflow are set to pressure-outlet. The other boundaries are set to wall. And no-slip boundary condition is selected at the wall.

In the following procedures including PBD, steepest ascent design and CCF design, the OCU is obtained by using this numerical simulation method. The calculation work is performed on a workstation (Gistom W510) whose CPU is Xeon E5645 with speed of $2.40 \mathrm{GHz}$.

\subsection{Laboratory experiment}

The downhole oil-water separation process consists of extraction, separation, lifting and injection. In this experiment, only the separation process is considered to test the separation performance of downhole oil-water separator. Therefore, an experimental joint was specially designed for this experiment (Fig. 4a). The work flow of 
the system is as follows: the liquid from the pump passes through flow channel g (Fig. 1) into the first DOHC. After the first separation, the oil-rich liquid flows back to the oil tank through the first overflow channel and pipes; the water-rich liquid passes through channel $\mathrm{h}$ into the second DOHC. After the second separation, the oil-rich liquid passes through the second overflow channel and pipes into the oil tank; the liquid with trace oil flows back to the oil tank.

Globe valve $\mathrm{V}_{2}, \mathrm{~V}_{3}, \mathrm{~V}_{4}$ are used to control the outlet pressures of the first overflow, second overflow and underflow for the adjustment of split ratio. Globe valve $\mathrm{V}_{1}$ is served to control the inlet flow rate. Sample valve $\mathrm{L}_{1}$,

$203 \mathrm{~L}_{2}, \mathrm{~L}_{3}, \mathrm{~L}_{4}$ are used for the collection of samples, including the inlet, first overflow, second overflow and underflow.

$204 \mathrm{P}_{1}, \mathrm{P}_{2}, \mathrm{P}_{3}$ and $\mathrm{P}_{4}$ represent pressure gauge; $\mathrm{Q}_{1}, \mathrm{Q}_{2}, \mathrm{Q}_{3}$ and $\mathrm{Q}_{4}$ represent turbine flowmeter. The centrifugal pump is adopted to drive the liquid in this experiment, and its flow rate can be controlled by variable frequency technique.

The following steps are proposed to prepare experimental media. Firstly, prepare the oil-water mixture with oil (diesel) concentration of $50000 \mathrm{mg} / \mathrm{L}$. Secondly, add OP-12 emulsifier to this mixture until its concentration is up to $15 \times 10^{-6} \mathrm{mg} / \mathrm{L}$. Finally, keep the blender running 70 seconds at the speed of $940 \mathrm{r} / \mathrm{min}$. The oil droplets size

210 detected by spectrophotometric method with the ultraviolet spectrophotometer (752C).

\section{3. Results and discussion}

212 3.1. Screening of significant factors using PBD

213 In this study, a 20 runs PBD was performed to evaluate the twelve factors at $95 \%$ confidence level. The effects 214 of each factor were evaluated at two levels, i.e. high level (+1) and low level (-1). Based on the results obtained by 215 our research group in previous studies, high level should be 1.25 2 times larger than low level (Zhao et al, 2014b). 216 The level of each factor is shown in Table 4. The factors and response (OCU) results of the PBD are shown in 217 Table5.

218 The standardized Pareto charts $(P<0.05)$ of main effects is depicted in Fig. 5. The critical values representing 219 the statistically significant effect of factors at $95 \%$ confidence level is 2.365 for the response. It illustrates that 220 overflow diameter $D_{\mathrm{o} 1}(\mathrm{~A})$, large cone angle $\alpha_{2}(\mathrm{I})$, tail pipe diameter $D_{\mathrm{u} 2}(\mathrm{~K})$ and tail pipe length $L_{\mathrm{u} 2}(\mathrm{~L})$ have 221 positive effect on OCU, and the other factors have negative effect on it. The significance of each parameter is evaluated by standardized main effect. The effects of the twelve factors on reducing OCU is in the following order: $\mathrm{J}>\mathrm{D}>\mathrm{C}>\mathrm{I}>\mathrm{K}>\mathrm{L}>\mathrm{F}>\mathrm{H}>\mathrm{G}>\mathrm{A}>\mathrm{E}>\mathrm{B}$. The small cone angle $\beta_{2}(\mathrm{~J})$, small cone angle $\beta_{1}(\mathrm{D})$, large cone angle $\alpha_{1}(\mathrm{C})$, 
224 large cone angle $\alpha_{2}$ (I) and tail pipe diameter $D_{\mathrm{u} 2}(\mathrm{~K})$ are considered as significant factors and included for the next 225 stage of optimization, because their standardized main effect are not less than 2.365 .

226 3.2. Determination of the central level for CCF design by steepest ascent design

227 The steepest ascent path of the significant factor was determined by the PBD results. Due to the large cone 228 angle $\alpha_{2}$ and the tail pipe diameter $D_{\mathrm{u} 2}$ having positive effects on OCU, their values should be increased. While the 229 small cone angle $\beta_{2}$, small cone angle $\beta_{1}$ and large cone angle $\alpha_{1}$ having negative effects on OCU, their values 230 should be reduced. The values of other factors keep the same as listed in Table 1. The steepest ascent design and 231 results are shown in Table 6. It shows that the OCU gradually decreases from experiment 1 to experiment 3; the 232 OCU tends to gradually increase in experiment 4. According to the principle of steepest ascent (Dong et al., 2003; 233 Yao et al., 2013), the minimum OCU may be between experiment 3 and experiment 4 . Thus, the parameters of 234 experiment 3 are used as the central level of the next response surface design.

235 3.3. Establishment of the equation for OCU using RSM

236 In the CCF design, the effect of each factor was evaluated at three levels, low level (-1), central level (0) and 237 high level (+1). The central level of the five factors is from the parameters of experiment 3 in steepest ascent design, 238 the low level and high level are shown in Table 7. The factors and response results of the CCF design are shown in 239 Table 8.

240 The equation for OCU $(Y)$ was obtained by performing multiple regression analysis, which could be written 241 as:

$$
Y(\mathrm{OCU})=2136.820-287.955 X_{1}-401.283 X_{2}-43.765 X_{3}-29.417 X_{4}-210.282 X_{5}
$$$$
+87.295 X_{1}^{2}+111.295 X_{2}^{2}+1.456 X_{3}^{2}+1.706 X_{4}^{2}+23.122 X_{5}^{2}+52.125 X_{1} X_{2}
$$$$
+7.406 X_{1} X_{3}-5.156 X_{1} X_{4}-12.604 X_{1} X_{5}+4.406 X_{2} X_{3}-3.906 X_{2} X_{4}
$$

243 Where $X_{1}, X_{2}, X_{3}, X_{4}$ and $X_{5}$ are given by Table 7.

244 The adequacy of the regression model was checked using ANOVA, as shown in Table 9. Significance of the 245 five factors and their interactions in the observed model were checked using the $F$-test. The Model $F$-value of 246224.66 implies the model is significant. There is only a $0.01 \%$ chance that a "model $F$-value" this large can occur 247 due to noise. Values of "Prob $>F$ " less than 0.05 indicate model terms are significant. In this case, linear terms $X_{1}$, $248 X_{2}, X_{3}, X_{4}, X_{5}$, and quadratic terms $X_{1}^{2}$ and $X_{2}^{2}$ are significant for OCU. Interactive terms are also significant for 249 OCU except $X_{3} X_{5}$ and $X_{4} X_{5}$. 
The $R^{2}$ value of the regression equation is 0.993 indicating that the model is fit to explain OCU as a function

of the five factors. The value of adjusted $R^{2}$ is 0.989 , which indicates that only $1.1 \%$ of the variations can not be explained by this model.

\section{3.4. Optimization of significant factors by PSO algorithm}

The levels of the five factors were taken at a central point of coded value zero for the convenience of

programming and calculation. Thus, the coded levels of the five factors were in $[-1,1]$. If $x_{i}$ is the coded level of a

factor, and $X_{i}$ is the true level of a factor, the relationship between them is:

$x_{i}=\left(X_{i}-X_{i 0}\right) / \Delta X_{i}$

Where $X_{i 0}$ is the true value of central level; $\Delta X_{i}$ is half of the difference between high and low levels.

The regression equation between the factors (in coded levels) and OCU $(y)$ could be written as:

$y(\mathrm{OCU})=154.636+66.029 x_{1}+40.324 x_{2}+19.441 x_{3}-19.029 x_{4}-12.471 x_{5}+21.824 x_{1}^{2}$

$+27.824 x_{2}^{2}+5.824 x_{3}^{2}+6.824 x_{4}^{2}+8.324 x_{5}^{2}+13.031 x_{1} x_{2}+7.406 x_{1} x_{3}-5.156 x_{1} x_{4}$

$-3.781 x_{1} x_{5}+4.406 x_{2} x_{3}-3.906 x_{2} x_{4}-3.656 x_{2} x_{5}-4.531 x_{3} x_{4}-1.406 x_{3} x_{5}-1.844 x_{4} x_{5}$

Based on the theory of PSO algorithm, a calculation program was compiled by the aid of Matlab software

(version 7.0) to search the optimal solution. The parameters setting of PSO algorithm are shown in Table 10. The

fitness curve of solution process is shown in Fig. 6. The optimized solutions and corresponding true values are listed in Table 11.

\subsection{Comparative analysis of structural parameters before and after optimization}

Compared with initial structure parameters, five structural parameters have changed after optimization. As

shown in Fig. 7, the small cone angles $\beta_{1}, \beta_{2}$ and the large cone angle $\alpha_{1}$ decrease, while the large cone angle $\alpha_{2}$ and the tail pipe diameter $D_{\mathrm{u} 2}$ increase.

The reduction of the large cone angle $\alpha_{1}$ and small cone angles $\beta_{1}$ of the first DOHC can increase the residence time of oil droplets without damping the swirling stream. Therefore, more oil droplets will be separated with full radial migration time thus reducing the OCU. Furthermore, it can be seen from the response surface plot and corresponding contour plot as shown in Fig. 8 that in a certain range, the smaller the large cone angle $\alpha_{1}$ and the smaller the cone angle $\beta_{1}$, the lower is the OCU, as expected.

The reductive amplitude of the small cone angle $\beta_{2}$ is larger than that of the small cone angle $\beta_{1}$. If the small 
276 centrifugal field and the increase of OCU. However, due to the increase of large cone angle $\alpha_{2}$, the large cone 277 section becomes steeper. And the rotary speed of the fluid entering into the small section will be accelerated, which makes up for the reduction of centrifugal field intensity in the small section and enhances the chance of separating more droplets. Moreover, it can be noticed from Fig. 9 that the optimal OCU will be observed when the small cone angle $\beta_{2}$ is small and the large cone angle $\alpha_{2}$ is large. Besides, the increase of tail pipe diameter $D_{\mathrm{u} 2}$ avoids a too

281 long small cone section in the second DOHC, and ensures the centrifugal field intensity in the small cone section is strong enough. And as shown in Fig. 10, with the reduction of the small cone angle $\beta_{2}$ and the increase of the tail

283 pipe diameter $D_{\mathrm{u} 2}$, the OCU gradually decreases in a certain range.

\section{3.6. Comparative analysis of separation performance before and after optimization}

The OCUs of original and optimized geometries were calculated by the numerical simulation method in

Section 2.4. In this simulation, in order to compare the separation performance with different oil droplets sizes, the oil droplets size distribution including six droplet sizes as shown in Fig. 11b was used in algebraic slip mixture model, which was obtained by dividing the measured oil droplets size distribution of the inlet stream (Fig. 11a) into six groups and summing the corresponding volume fractions. Thus, the phase number of the model should be set to7 including a water phase and six kinds of oil phases. The particle sizes $(d)$ of the six kinds of oil phases are also shown in Fig. 11b. geometry and the best geometry in CCF design (experiment 51 in Table 8), the OCU of the optimized geometry is reduced by $52.4 \%$ and $16 \%$, respectively.

The oil phase volume fraction of the optimized geometry in yoz cross section is shown in Fig. 12. It can be seen clearly from the figure, for the oil phase of $d=6.06 \mu \mathrm{m}$, the oil core can be found in the two DOHCs. Besides the oil core, the oil phase in other areas is still visible. The oil concentration in the tail pipe of the second DOHC is obviously less than that of the first DOHC. For the oil phase of $d=14.63 \mu \mathrm{m}$, the oil core also can be found in both of the two DOHCs. But the oil core concentration of the first DOHC, is better than that of the second DOHC. And the oil concentration is begin to greatly reduce except the oil core. For the oil phase of $d=25.31 \mu \mathrm{m}$, the oil core concentration of the second DOHC begins to weaken. For the oil phase of $d=35.78 \mu \mathrm{m}$, the oil core concentration of the first DOHC begins to weaken. And the oil core disappears from the small cone section of the second DOHC. 
304 oil phase of $d=168.95 \mu \mathrm{m}$, the oil core only exists in the first DOHC, but its concentration is lower than that of the 305 oil phase with $d=59.03 \mu \mathrm{m}$. With regard to the oil droplets whose particle sizes are $6.06 \mu \mathrm{m}, 14.63 \mu \mathrm{m}$ and $25.31 \mu \mathrm{m}$, which have not been fully separated in the first DOHC. Consequently, lots of oil droplets flow into the second DOHC for secondary separation, and oil cores were also formed in the second DOHC. As to the oil droplets whose particle sizes are $35.78 \mu \mathrm{m}, 59.03 \mu \mathrm{m}$ and $168.95 \mu \mathrm{m}$, since most of the oil droplets have been separated in the first

DOHC, no obvious oil core can be found in the second DOHC.

The OCU of each particle size for the original and optimized geometries is shown in Fig. 13. It can be seen that for the oil droplets whose particle sizes are not smaller than $35.78 \mu \mathrm{m}$, the OCU decreases slightly after optimization. However, for the oil droplets whose particle sizes are smaller than $35.78 \mu \mathrm{m}$, the OCU reduces greatly.

313 Therefore, the separation performance of the optimized geometry is remarkably improved for the oil droplets with 314 small particle size.

\section{3.7. Validation of laboratory experiment}

316 In order to validate the effectiveness of the proposed optimization method, laboratory experiments were carried out. The physical parameters of the experimental media can be in agreement with that of the numerical 318 simulation by using the method in Section 2.5 to prepare experimental media. The outlet pressures of overflow and 319 underflow can be in accordance with that of the numerical simulation by adjusting the globe valve $V_{1}, V_{2}, V_{3}$ and $320 \mathrm{~V}_{4}$. The OCUs obtained by the laboratory experiments are $223 \mathrm{mg} / \mathrm{L}$ (original geometry), $101 \mathrm{mg} / \mathrm{L}$ (optimized 321 geometry) and $121 \mathrm{mg} / \mathrm{L}$ (the best geometry in CCF design), respectively. Compared with original geometry and the 322 best geometry in CCF design, the OCU of the optimized geometry is reduced by $54.7 \%$ and $16.5 \%$, respectively.

323 On the basis of the above analyses, it can be summarized that the OCU of the optimized downhole oil-water separator significantly reduces. At the same time, a general agreement between the numerical result and experimental result is obtained. Therefore, the PSO algorithm combined with PBD, steepest ascent design and RSM can be an effective method for structural optimization of the downhole oil-water separator.

\section{Conclusions}

An optimization study was conducted in the downhole oil-water separator by using PSO algorithm combined with PBD, steepest ascent design and RSM in order to obtain a geometry that could minimize OCU of this separator. Before and after optimization, the variations of separation performance were investigated by CFD 
332 the following conclusions can be achieved:

333 (1) Small cone angle $\beta_{2}$, small cone angle $\beta_{1}$ and large cone angle $\alpha_{1}$ are significant factors having positive effect on OCU, while large cone angle $\alpha_{2}$ and tail pipe diameter $D_{\mathrm{u} 2}$ are significant factors having negative effect on OCU.

(2) The optimal structural parameters obtained are: the small cone angle $\beta_{2}$ is $1.5^{\circ}$, the small cone angle $\beta_{1}$ is $1.8^{\circ}$, the large cone angle $\alpha_{1}$ is $19.9^{\circ}$, the large cone angle $\alpha_{2}$ is $22.6^{\circ}$ and the tail pipe diameter $D_{\mathrm{u} 2}$ is $6.7 \mathrm{~mm}$. Compared with the original structural parameters, it is found that the variations of the structural parameters

\section{Acknowledgement}

The author would like to thank the finance supported by the National High Technology Research and

Development Program of China (No. 2006AA06Z224) and the Fundamental Research Funds for the Central

Universities (No.10CX05009A and No. 13CX06077A).

\section{References}

Amini, S., Mowla, D., Golkar, M., Esmaeilzadeh, F., 2012. Mathematical modelling of a hydrocyclone for the down-hole oil-water separation (dows). Chem. Eng. Res. Des. 90, 2186-2195.

Belaidi, A., Thew, M.T., 2003. The effect of oil and gas content on the controllability and separation in a de-oiling hydrocyclone. Chem. Eng. Res. Des. 81, 305-314.

Bergh, F.V.D., Engelbrecht, A.P., 2006. A study of particle swarm optimization particle trajectories. Inf. Sci. 176, 937-971.

Brennan, M., 2006. CFD simulations of hydrocyclones with an air core: comparison between large eddy simulations and a second moment closure. Chem. Eng. Res. Des. 84, 495-505. 
Chu, L.Y., Chen, W.M., Lee, X.Z., 2000. Effect of structural modification on hydrocyclone performance. Sep. Purif. Technol. 21, 71-86.

Delfos, R., Murphy, S., Stanbridge, D., Olujić, Ž., Jansens, P.J., 2004. A design tool for optimising axial

Dong, Y., Tang, J., Xu, B., Wang, D., 2005. An application of swarm optimization to nonlinear programming. Comput. Math. Appl. 49, 1655-1668.

Grady, S.A., Wesson, G.D., Abdullah, M., Kalu, E.E., 2003. Prediction of 10-mm hydrocyclone separation efficiency using computational fluid dynamics. Filtr. Sep. 40, 41-46.

Ghodrat, M., Kuang, S.B., Yu, A.B., Vince, A., Barnett, G.D., Barnett, P.J., 2014a. Numerical analysis of hydrocyclones with different conical section designs. Miner. Eng. 62, 74-84.

Ghodrat, M., Kuang, S.B., Yu, A.B., Vince, A., Barnett, G.D., Barnett, P.J., 2014b. Numerical analysis of hydrocyclones with different vortex finder configurations. Miner. Eng. 63, 125-138.

Joyce, A.P., Leung, S.S., 2013. Use of response surface methods and path of steepest ascent to optimize ligand-binding assay sensitivity. J. Immunol. Methods. 392, 12-13.

Karami, H.R., Keyhani, M., Mowla, D., 2016. Experimental analysis of drag reduction in the pipelines with response surface methodology. J. Pet. Sci. Eng. 138, 104-112.

Kharoua, N., Khezzar, L., Nemouchi, Z., 2010. Hydrocyclones for De-oiling Applications-A Review. Petrol. Sci. Technol. 28, 735-755.

Li, D., Zhou X., Zhou, H., 2011a. Experimental Study on Inlet Structure of the Rod Pump with Down-hole Oil-water Hydrocyclone. Procedia Eng. 18, 369-374.

Li, D., Zhou X., Chen, Y., 2011b. Experimental Study on Overflow Pipe Structure of the Rod Pump with Down-Hole Oil-Water Hydrocyclone. Procedia Eng. 18, 387-391.

Mangili, I., Lasagni, M., Huang, K., Isayev, A.I., 2015. Modeling and optimization of ultrasonic devulcanization using the response surface methodology based on central composite face-centered design. Chemom. Intell. Lab. Syst. 144, 1-10.

Noroozi, S., Hassan, S., Hashemabadi, S.H., 2009. CFD simulation of inlet design effect on de-oiling hydrocyclone separation efficiency. Chem. Eng. Technol. 32, 1885-1893.

Noroozi, S., Hashemabadi, S.H., 2011. CFD analysis of inlet chamber body profile effects on de-oiling 
hydrocyclone efficiency. Chem. Eng. Res. Des. 89, 968-977.

Ogunsina, O.O., Wiggins, M.L., 2005. A review of downhole separation technology. In: SPE Production and Operations Symposium. Society of Petroleum Engineers.

Priyadharshini, S. D., Bakthavatsalam, A. K., 2016. Optimization of phenol degradation by the microalga chlorella pyrenoidosa using placket-burman design and response surface methodology. Bioresour. Technol. 207, $150-156$.

Rao, R.V., Patel, V.K., 2010. Thermodynamic optimization of cross flow plate-fin heat exchanger using a particle swarm optimization algorithm. Int. J. Therm. Sci. 49, 1712-1721.

Saidi, M., Maddahian, R., Farhanieh, B., 2013. Numerical investigation of cone angle effect on the flow field and separation efficiency of deoiling hydrocyclones. Heat Mass Transfer. 49,247-260.

Scaramuzza, J.L., Fischetti, H., Strappa, L., Figliuolo, S., 2001. Downhole Oil/Water Separation System-Field Pilot-Secondary Recovery Application Project. In: SPE Latin American and Caribbean Petroleum Engineering Conference. Society of Petroleum Engineers.

Shi, Y., Eberhart, R.C., 1998. Parameter Selection in Particle Swarm Optimization. Proceedings of the 7th International Conference on Evolutionary Programming VII. Springer-Verlag.

Silva, D.O., Vieira, L.G.M., Lobato, F.S., Barrozo, M.A.S., 2012. Optimization of the design and performance of hydrocyclones by differential evolution technique. Chem. Eng. Process. 61, 1-7.

Silva, N.K.G., Silva, D.O., Vieira, L.G.M., Barrozo, M.A.S., 2015. Effects of underflow diameter and vortex finder length on the performance of a newly designed filtering hydrocyclone. Powder Technol. 286, 305-310.

Šereš, Z., Maravić, N., Takači, A., Nikolić, I., Šoronja-Simović, D., Jokić, A., Hodur, C., 2016. Treatment of vegetable oil refinery wastewater using alumina ceramic membrane: optimization using response surface methodology. J. Cleaner Prod. 112, 3132-3137.

Thew, M.T., 1986. Hydrocyclone redesign for liquid-liquid separation. Chem. Eng. 427: 17-23.

Thew, M.T., 2000. Flotation | cyclones for oil/water separations. In: Wilson,I.D.(Ed.), Encylopedia of Separation Science. Academic Press.1480-1490

Vieira, L.G.M., Silvério, B.C., Damasceno, J.J.R., Barrozo, M.A.S., 2011. Performance of hydrocyclones with

415 Yao, C., Liu, C., Chu, I., Hsieh, T., Hwang, S., 2003. Factorial designs combined with the steepest ascent method to 
optimize serum-free media for ex vivo expansion of human hematopoietic progenitor cells. Comput. Enzyme Micro. Tech. 33, 343-352.

418 Young, G.A.B., Wakley, W.D., Taggart, D.L., Andrews, S.L., Worrell, J.R., 1994. Oil-water separation using 419 hydrocyclones-An experimental search for optimum dimensions. J. Petrol. Sci. Eng. 11, 37-50.

420 Zhao, C., Li, Z., 2011. Design of down-hole oil-water separation and reinjection in the same well system with $421 \quad$ surface driving single screw pump. Adv. Mater. Res. 339, 595-602.

422 Zhao, C., Li, Z., Dong, X., 2013. Design and ground test of downhole oil-water separation system with screw pump. 423 Zhongguo Shiyou Daxue Xuebao. 37, 129-133. In Chinese.

424 Zhao, C., Li, Z., Dong, X., Zhang, X., 2014a. Numerical simulation and experiment of downhole two-stage tandem hydrocyclone. Acta Petrolei Sinica. 3, 551-557. In Chinese.

Zhao, C, Li, Z., Xu, W., Wu, H., 2014b. Structural parameter optimization of downhole dual-stage tandem hydrocyclone. Chemical Engineering. 42, 48-53. In Chinese.

\section{Tables}

Table 1 Structural parameters of the downhole oil-water separator obtained from previous studies.

\begin{tabular}{ccc}
\hline Parameters & The first DOHC & The second DOHC \\
\hline Nominal diameter $D(\mathrm{~mm})$ & 18 & 13 \\
Cylindrical length $L_{\mathrm{c}}(\mathrm{mm})$ & 36 & 26 \\
Large cone angle $\alpha_{1}\left(^{\circ}\right)$ & 22 & 22 \\
Small cone angle $\beta_{1}\left({ }^{\circ}\right)$ & 2 & 2 \\
Tail pipe diameter $D_{\mathrm{u}}(\mathrm{mm})$ & 9.0 & 6.5 \\
Tail pipe length $L_{\mathrm{u}}(\mathrm{mm})$ & 360 & 260 \\
Overflow diameter $D_{\mathrm{o}}(\mathrm{mm})$ & 3 & 2.5 \\
Equivalent inlet diameter $D_{\mathrm{i}}(\mathrm{mm})$ & 4.96 & 4.43 \\
Inlet length $D_{\mathrm{ik}}(\mathrm{mm})$ & 8.1 & 7.3 \\
Inlet width $D_{\mathrm{ic}}(\mathrm{mm})$ & 2.4 & 2.1 \\
\hline
\end{tabular}

Table 2 Physical Parameters of materials.

\begin{tabular}{cccc}
\hline Material & Density $\left(\mathrm{kg} / \mathrm{m}^{3}\right)$ & Viscosity $(\mathrm{Pa} \bullet \mathrm{s})$ & Median particle size $(\mu \mathrm{m})$ \\
\hline Water & 997 & $8.94 \times 10^{-4}$ & - \\
Oil & 868 & $3.46 \times 10^{-3}$ & 30 \\
\hline
\end{tabular}

Table 3 Setting of boundary condition.

\begin{tabular}{ccc}
\hline Parameters & Value & Unit \\
\hline Velocity inlet & 0.73 & $\mathrm{~m} / \mathrm{s}$ \\
Oil volume fraction of entrance & 0.0576 & - \\
Hydraulic diameter & 0.012 & $\mathrm{~m}$
\end{tabular}




\begin{tabular}{ccc} 
Turbulence intensity & 0.047 & - \\
Pressure outlet(overflow) & 15.6 & $\mathrm{MPa}$ \\
Pressure outlet(underflow) & 16 & $\mathrm{MPa}$ \\
\hline
\end{tabular}

Table 4 Factors and levels in PBD.

\begin{tabular}{cccc}
\hline \multirow{2}{*}{ Factors } & Symbol & \multicolumn{2}{c}{ Level } \\
\cline { 3 - 4 } & & Low $(-1)$ & High $(+1)$ \\
\hline Overflow diameter $D_{\mathrm{o} 1}(\mathrm{~mm})$ & $\mathrm{A}$ & 2.5 & 3.75 \\
Cylindrical length $L_{\mathrm{c} 1}(\mathrm{~mm})$ & $\mathrm{B}$ & 28.8 & 43.2 \\
Large cone angle $\alpha_{1}\left(^{\circ}\right)$ & $\mathrm{C}$ & 17 & 25 \\
Small cone angle $\beta_{1}\left(^{\circ}\right)$ & $\mathrm{D}$ & 1.5 & 3 \\
Tail pipe diameter $D_{\mathrm{u} 1}(\mathrm{~mm})$ & $\mathrm{E}$ & 7.2 & 10.8 \\
Tail pipe length $L_{\mathrm{u} 1}(\mathrm{~mm})$ & $\mathrm{F}$ & 315 & 405 \\
Overflow diameter $D_{\mathrm{o} 2}(\mathrm{~mm})$ & $\mathrm{G}$ & 2 & 3 \\
Cylindrical length $L_{\mathrm{c} 2}(\mathrm{~mm})$ & $\mathrm{H}$ & 20.8 & 31.2 \\
Large cone angle $\alpha_{2}\left(^{\circ}\right)$ & $\mathrm{I}$ & 17 & 25 \\
Small cone angle $\beta_{2}\left(^{\circ}\right)$ & $\mathrm{J}$ & 1.5 & 3 \\
Tail pipe diameter $D_{\mathrm{u} 2}(\mathrm{~mm})$ & $\mathrm{K}$ & 5.2 & 7.8 \\
Tail pipe length $L_{\mathrm{u} 2}(\mathrm{~mm})$ & $\mathrm{L}$ & 227.5 & 292.5 \\
\hline
\end{tabular}

Table 5 Factors and response results of PBD.

\begin{tabular}{|c|c|c|c|c|c|c|c|c|c|c|c|c|c|}
\hline \multirow{2}{*}{ Run order } & \multicolumn{12}{|c|}{ Factors } & \multirow{2}{*}{ OCU $(\mathrm{mg} / \mathrm{L})$} \\
\hline & $\mathrm{A}$ & B & $\mathrm{C}$ & $\mathrm{D}$ & $\mathrm{E}$ & $\mathrm{F}$ & G & $\mathrm{H}$ & I & $\mathrm{J}$ & $\mathrm{K}$ & $\mathrm{L}$ & \\
\hline 1 & -1 & -1 & 1 & 1 & -1 & 1 & 1 & -1 & -1 & -1 & -1 & 1 & 270 \\
\hline 2 & -1 & 1 & -1 & 1 & 1 & 1 & 1 & -1 & -1 & 1 & 1 & -1 & 286 \\
\hline 3 & -1 & -1 & -1 & -1 & 1 & -1 & 1 & -1 & 1 & 1 & 1 & 1 & 566 \\
\hline 4 & 1 & -1 & 1 & 1 & -1 & -1 & -1 & -1 & 1 & -1 & 1 & -1 & 434 \\
\hline 5 & 1 & 1 & -1 & -1 & 1 & 1 & -1 & 1 & 1 & -1 & -1 & -1 & 538 \\
\hline 6 & -1 & -1 & 1 & -1 & 1 & -1 & 1 & 1 & 1 & 1 & -1 & -1 & 352 \\
\hline 7 & 1 & -1 & 1 & 1 & 1 & 1 & -1 & -1 & 1 & 1 & -1 & 1 & 330 \\
\hline 8 & 1 & -1 & -1 & 1 & 1 & -1 & 1 & 1 & -1 & -1 & -1 & -1 & 455 \\
\hline 9 & -1 & 1 & 1 & 1 & 1 & -1 & -1 & 1 & 1 & -1 & 1 & 1 & 539 \\
\hline 10 & 1 & 1 & 1 & -1 & -1 & 1 & 1 & -1 & 1 & 1 & -1 & -1 & 393 \\
\hline 11 & 1 & 1 & -1 & 1 & 1 & -1 & -1 & -1 & -1 & 1 & -1 & 1 & 301 \\
\hline 12 & 1 & -1 & -1 & -1 & -1 & 1 & -1 & 1 & -1 & 1 & 1 & 1 & 495 \\
\hline 13 & -1 & 1 & 1 & -1 & -1 & -1 & -1 & 1 & -1 & 1 & -1 & 1 & 285 \\
\hline 14 & -1 & 1 & 1 & -1 & 1 & 1 & -1 & -1 & -1 & -1 & 1 & -1 & 506 \\
\hline 15 & -1 & 1 & -1 & 1 & -1 & 1 & 1 & 1 & 1 & -1 & -1 & 1 & 503 \\
\hline 16 & -1 & -1 & -1 & -1 & -1 & -1 & -1 & -1 & -1 & -1 & -1 & -1 & 596 \\
\hline 17 & 1 & -1 & 1 & -1 & 1 & 1 & 1 & 1 & -1 & -1 & 1 & 1 & 467 \\
\hline 18 & 1 & 1 & 1 & 1 & -1 & -1 & 1 & 1 & -1 & 1 & 1 & -1 & 213 \\
\hline
\end{tabular}




\begin{tabular}{cccccccccccccc}
19 & -1 & -1 & -1 & 1 & -1 & 1 & -1 & 1 & 1 & 1 & 1 & -1 & 419 \\
20 & 1 & 1 & -1 & -1 & -1 & -1 & 1 & -1 & 1 & -1 & 1 & 1 & 791 \\
\hline
\end{tabular}

Table 6 Steepest ascent design and results.

\begin{tabular}{ccccccc}
\hline Run order & $\begin{array}{r}\text { Small cone } \\
\text { angle } \beta_{2}\left(^{\circ}\right)\end{array}$ & $\begin{array}{c}\text { Small cone } \\
\text { angle } \beta_{1}\left({ }^{\circ}\right)\end{array}$ & $\begin{array}{c}\text { Large cone } \\
\text { angle } \alpha_{1}\left(^{\circ}\right)\end{array}$ & $\begin{array}{c}\text { Large cone } \\
\text { angle } \alpha_{2}\left({ }^{\circ}\right)\end{array}$ & $\begin{array}{c}\text { Tail pipe } \\
\text { diameter } \\
D_{\mathrm{u} 2}(\mathrm{~mm})\end{array}$ & OCU(mg/L) \\
\hline 1 & 3 & 3 & 25 & 17 & 5.2 & 264 \\
2 & 2.5 & 2.5 & 23 & 19 & 5.8 & 203 \\
3 & 2 & 2 & 21 & 21 & 6.4 & 7 \\
4 & 1.5 & 1.5 & 19 & 23 & 7.6 & 219 \\
5 & 1 & 1 & 17 & 25 & 7.6 & 257 \\
\hline
\end{tabular}

Table 7 Factors and levels of CCF design.

\begin{tabular}{ccccc}
\hline \multirow{2}{*}{ Factors } & Symbol & \multicolumn{3}{c}{ level } \\
\cline { 3 - 5 } & & Central (0) & Low (-1) & High (+1) \\
\hline Small cone angle $\beta_{2}\left({ }^{\circ}\right)$ & $X_{1}$ & 2 & 1.5 & 2.5 \\
Small cone angle $\beta_{1}\left(^{\circ}\right)$ & $X_{2}$ & 2 & 1.5 & 2.5 \\
Large cone angle $\alpha_{1}\left(^{\circ}\right)$ & $X_{3}$ & 21 & 19 & 23 \\
Large cone angle $\alpha_{2}\left({ }^{\circ}\right)$ & $X_{4}$ & 21 & 19 & 23 \\
Tail pipe diameter $D_{\mathrm{u} 2}(\mathrm{~mm})$ & $X_{5}$ & 6.4 & 5.8 & 7 \\
\hline
\end{tabular}

Table 8 Factors and response results of CCF design.

\begin{tabular}{|c|c|c|c|c|c|c|}
\hline \multirow{2}{*}{ Run order } & \multicolumn{5}{|c|}{ Factors } & \multirow{2}{*}{$\mathrm{OCU}(\mathrm{mg} / \mathrm{L})$} \\
\hline & $X_{1}$ & $X_{2}$ & $X_{3}$ & $X_{4}$ & $X_{5}$ & \\
\hline 1 & 0 & 1 & 0 & 0 & 0 & 217 \\
\hline 2 & 0 & 0 & 0 & 0 & 0 & 155 \\
\hline 3 & 0 & 0 & 1 & 0 & 0 & 174 \\
\hline 4 & -1 & -1 & -1 & -1 & -1 & 132 \\
\hline 5 & -1 & 1 & -1 & -1 & 1 & 177 \\
\hline 6 & -1 & 1 & 1 & -1 & -1 & 233 \\
\hline 7 & -1 & -1 & -1 & 1 & -1 & 113 \\
\hline 8 & -1 & 1 & -1 & 1 & 1 & 150 \\
\hline 9 & 0 & -1 & 0 & 0 & 0 & 147 \\
\hline 10 & 1 & -1 & -1 & -1 & -1 & 241 \\
\hline 11 & 1 & -1 & 1 & 1 & -1 & 246 \\
\hline 12 & -1 & 1 & 1 & 1 & 1 & 147 \\
\hline 13 & 0 & 0 & 0 & 0 & 0 & 155 \\
\hline 14 & 1 & 1 & 1 & 1 & -1 & 367 \\
\hline 15 & 1 & 1 & 1 & 1 & 1 & 332 \\
\hline 16 & -1 & -1 & 1 & 1 & -1 & 134 \\
\hline 17 & 0 & 0 & 0 & 0 & 0 & 155 \\
\hline 18 & 0 & 0 & 0 & 1 & 0 & 148 \\
\hline
\end{tabular}




\begin{tabular}{|c|c|c|c|c|c|c|}
\hline 19 & 1 & 1 & 1 & -1 & 1 & 395 \\
\hline 20 & 0 & 0 & 0 & 0 & 0 & 155 \\
\hline 21 & 0 & 0 & 0 & 0 & 0 & 155 \\
\hline 22 & -1 & 1 & 1 & -1 & 1 & 212 \\
\hline 23 & 0 & 0 & 0 & -1 & 0 & 174 \\
\hline 24 & 0 & 0 & 0 & 0 & 0 & 155 \\
\hline 25 & 0 & 0 & -1 & 0 & 0 & 146 \\
\hline 26 & 1 & 1 & 1 & -1 & -1 & 435 \\
\hline 27 & 0 & 0 & 0 & 0 & 0 & 155 \\
\hline 28 & -1 & 1 & -1 & 1 & -1 & 175 \\
\hline 29 & 1 & 0 & 0 & 0 & 0 & 230 \\
\hline 30 & -1 & 1 & 1 & 1 & -1 & 200 \\
\hline 31 & -1 & -1 & 1 & -1 & 1 & 154 \\
\hline 32 & 1 & 1 & -1 & 1 & 1 & 266 \\
\hline 33 & 1 & -1 & 1 & 1 & 1 & 214 \\
\hline 34 & 0 & 0 & 0 & 0 & 0 & 155 \\
\hline 35 & 0 & 0 & 0 & 0 & 0 & 155 \\
\hline 36 & -1 & -1 & 1 & -1 & -1 & 158 \\
\hline 37 & 1 & -1 & -1 & -1 & 1 & 230 \\
\hline 38 & 1 & 1 & -1 & 1 & -1 & 304 \\
\hline 39 & 0 & 0 & 0 & 0 & 1 & 155 \\
\hline 40 & 1 & -1 & -1 & 1 & 1 & 186 \\
\hline 41 & -1 & 0 & 0 & 0 & 0 & 122 \\
\hline 42 & 1 & 1 & -1 & -1 & 1 & 317 \\
\hline 43 & -1 & -1 & -1 & -1 & 1 & 121 \\
\hline 44 & -1 & 1 & -1 & -1 & -1 & 195 \\
\hline 45 & 1 & -1 & 1 & -1 & -1 & 301 \\
\hline 46 & 0 & 0 & 0 & 0 & 0 & 155 \\
\hline 47 & 1 & 1 & -1 & -1 & -1 & 350 \\
\hline 48 & 1 & -1 & 1 & -1 & 1 & 264 \\
\hline 49 & 0 & 0 & 0 & 0 & -1 & 170 \\
\hline 50 & -1 & -1 & 1 & 1 & 1 & 129 \\
\hline 51 & -1 & -1 & -1 & 1 & 1 & 106 \\
\hline 52 & 1 & -1 & -1 & 1 & -1 & 225 \\
\hline
\end{tabular}

Table 9 ANOVA for multiple regression model.

\begin{tabular}{cccccc}
\hline Source & Df & Sum of squares & Mean square & $F$-value & $\begin{array}{c}P \text {-value } \\
\text { Prob }>F\end{array}$ \\
\hline Model & 20 & 296353 & 14818 & 224.66 & $<0.0001$ \\
Linear & 5 & 2045 & 46794 & 709.48 & $<0.0001$ \\
$X_{1}$ & 1 & 148236 & 148236 & 2247.54 & $<0.0001$ \\
$X_{2}$ & 1 & 55284 & 55284 & 838.20 & $<0.0001$ \\
$X_{3}$ & 1 & 12851 & 12851 & 194.84 & $<0.0001$
\end{tabular}




\begin{tabular}{|c|c|c|c|c|c|}
\hline$X_{4}$ & 1 & 12312 & 12312 & 186.67 & $<0.0001$ \\
\hline$X_{5}$ & 1 & 5288 & 5288 & 80.17 & $<0.0001$ \\
\hline Square & 5 & 51520 & 10304 & 156.23 & $<0.0001$ \\
\hline$X_{1}^{2}$ & 1 & 45719 & 1179 & 17.88 & $<0.0001$ \\
\hline$X_{2}^{2}$ & 1 & 5102 & 1917 & 29.06 & $<0.0001$ \\
\hline$X_{3}^{2}$ & 1 & 324 & 84 & 1.27 & 0.268 \\
\hline$X_{4}^{2}$ & 1 & 203 & 115 & 1.75 & 0.196 \\
\hline$X_{5}^{2}$ & 1 & 172 & 172 & 2.60 & 0.117 \\
\hline Interaction & 10 & 10864 & 1086 & 16.47 & $<0.0001$ \\
\hline$X_{1} X_{2}$ & 1 & 5434 & 5434 & 82.39 & $<0.0001$ \\
\hline$X_{1} X_{3}$ & 1 & 1755 & 1755 & 26.61 & $<0.0001$ \\
\hline$X_{1} X_{4}$ & 1 & 851 & 851 & 12.90 & 0.001 \\
\hline$X_{1} X_{5}$ & 1 & 458 & 458 & 6.94 & 0.013 \\
\hline$X_{2} X_{3}$ & 1 & 621 & 621 & 9.42 & 0.004 \\
\hline$X_{2} X_{4}$ & 1 & 488 & 488 & 7.40 & 0.011 \\
\hline$X_{2} X_{5}$ & 1 & 428 & 428 & 6.49 & 0.016 \\
\hline$X_{3} X_{4}$ & 1 & 657 & 657 & 9.96 & 0.004 \\
\hline$X_{3} X_{5}$ & 1 & 63 & 63 & 0.96 & 0.335 \\
\hline$X_{4} X_{5}$ & 1 & 109 & 109 & 1.65 & 0.209 \\
\hline Residual Error & 31 & 2045 & 66 & - & - \\
\hline Total & 51 & 298398 & - & - & - \\
\hline
\end{tabular}

Table 10 Parameters setting of PSO algorithm.

\begin{tabular}{cc}
\hline Parameters & Values \\
\hline Particle length & 5 \\
Particle rang & {$[-1,1]$} \\
Particle population size & 40 \\
Maximum velocity of a particle & 2 \\
Acceleration constants & $c_{1}=c_{2}=2$ \\
Maximum Iteration & 400 \\
\hline
\end{tabular}

Table 11 Optimized results of PSO algorithm.

\begin{tabular}{cccccc}
\hline \multirow{2}{*}{ Levels } & \multicolumn{5}{c}{ Factors } \\
\cline { 2 - 6 } & $\begin{array}{c}\text { Small cone } \\
\text { angle } \beta_{2}\left({ }^{\circ}\right)\end{array}$ & $\begin{array}{c}\text { Small cone } \\
\text { angle } \beta_{1}\left({ }^{\circ}\right)\end{array}$ & $\begin{array}{c}\text { Large cone } \\
\text { angle } \alpha_{1}\left({ }^{\circ}\right)\end{array}$ & $\begin{array}{c}\text { Large cone } \\
\text { angle } \alpha_{2}\left({ }^{\circ}\right)\end{array}$ & $\begin{array}{c}\text { Tail pipe } \\
\text { diameter } \\
D_{\mathrm{u} 2}(\mathrm{~mm})\end{array}$ \\
\hline Coded & -0.9975 & -0.3415 & -0.5524 & 0.8127 & 0.5013 \\
True & 1.5 & 1.8 & 19.9 & 22.6 & 6.7 \\
\hline
\end{tabular}

\section{Figures}

443 Fig. 1. Structure diagram of the downhole oil-water separator: 1-tubing; 2-rod; 3-tubing joint; 4-screw pump; 444 5-upper cross-channel nipple; 6-the first DOHC; 7-shell; 8-central cross-channel nipple; 9-the second 445 DOHC; 10 - under cross-channel nipple; 11 -inner pipe; 12 - outer pipe; a j-flow channel. 
446 Fig. 2. Inlet of the DOHC.

447 Fig. 3. Flow domain of the downhole oil-water separator.

448 Fig. 4. Flow diagram of experiment and experimental joint: (a) experimental joint and (b) flow diagram of 449 experiment.

450 Fig. 5. Standardized $(P<0.05)$ main effect Pareto charts of the PBD for OCU.

$451 \quad$ Fig. 6. Fitness curve of solution process.

452 Fig. 7. Variations of the five structural parameters before and after optimization.

453 Fig. 8. Surface and contour plot showing interactions between small cone angle $\beta_{1}$ and large cone angle $\alpha_{1}$ on OCU 454 while the other three factors at central level.

455 Fig. 9. Surface and contour plot showing interactions between small cone angle $\beta_{2}$ and large cone angle $\alpha_{2}$ on OCU 456 while the other three factors at central level.

457 Fig. 10. Surface and contour plot showing interactions between small cone angle $\beta_{2}$ and tail pipe diameter $D_{\mathrm{u} 2}$ on 458 OCU while the other three factors at central level.

459 Fig. 11. Oil droplet size distribution in inlet: (a) Actual measured distribution and (b) Simulation model 460 distribution.

461 Fig. 12. Contour of oil volume fraction.

462 Fig. 13. Oil concentrations in underflow of pre-optimization and post-optimization. 

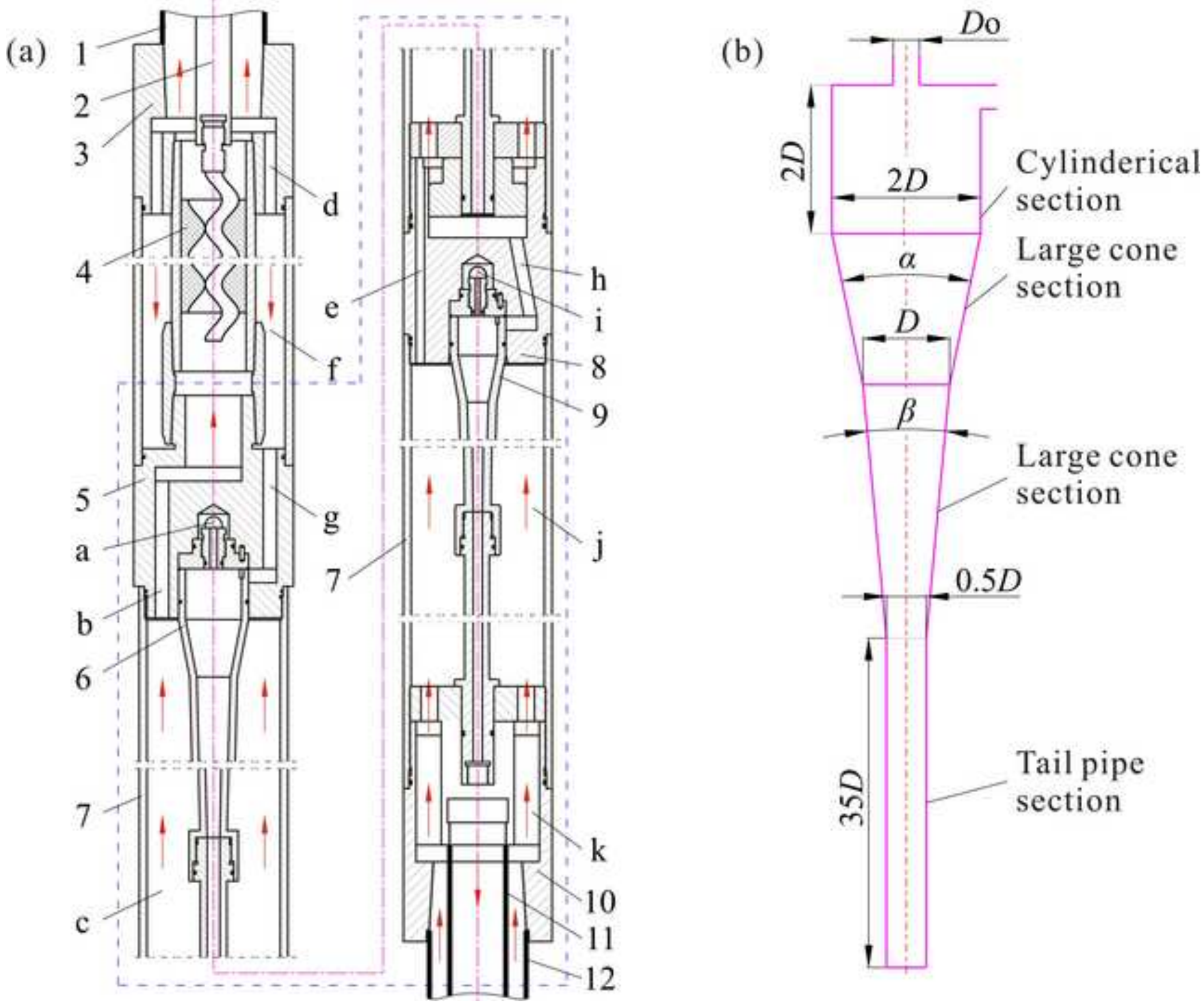


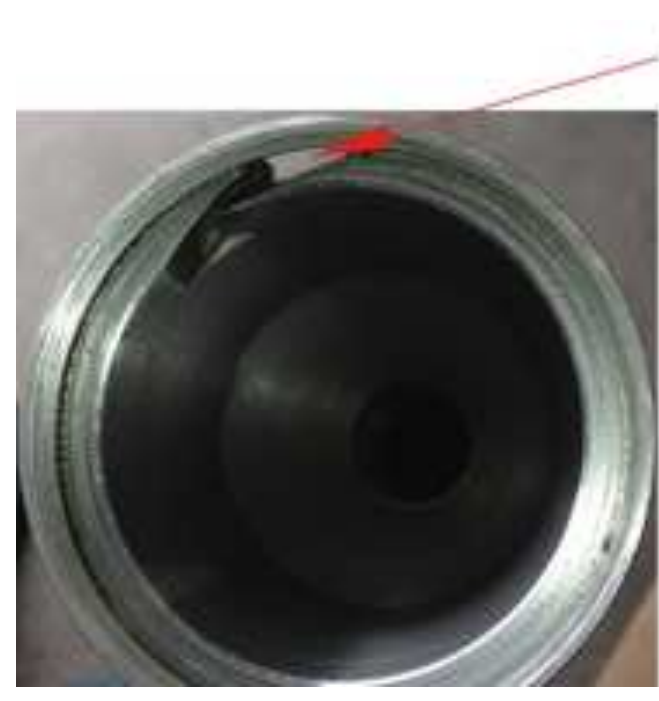

Inlet

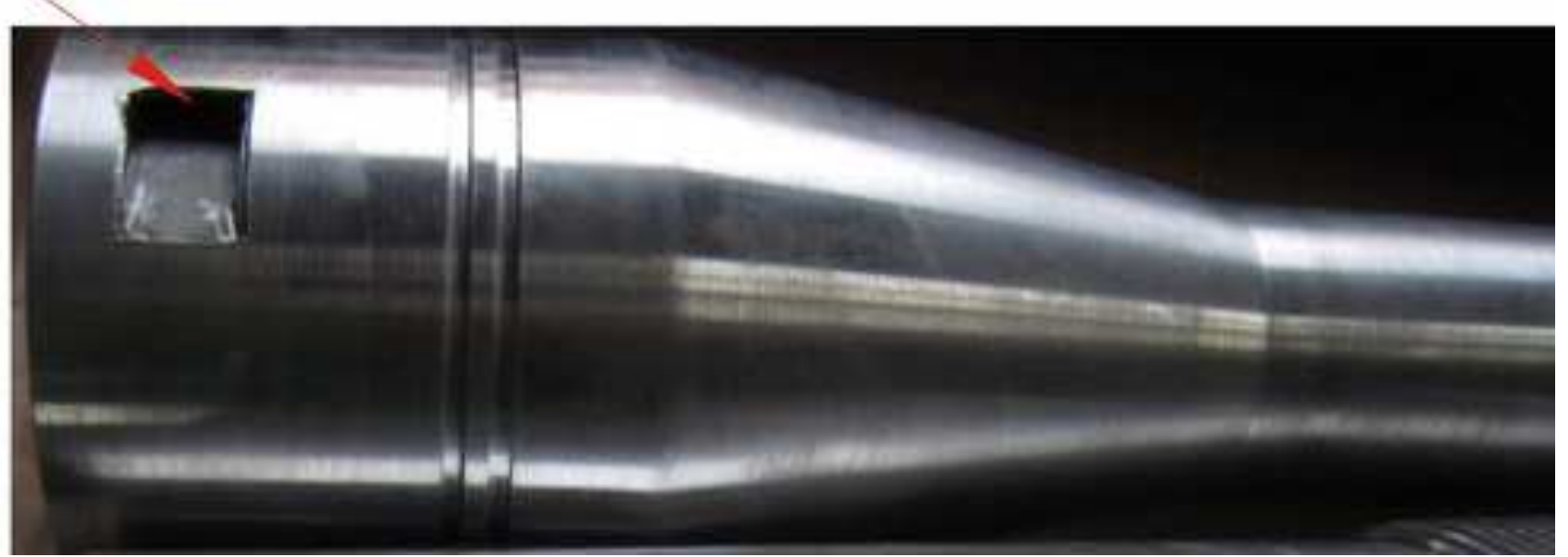



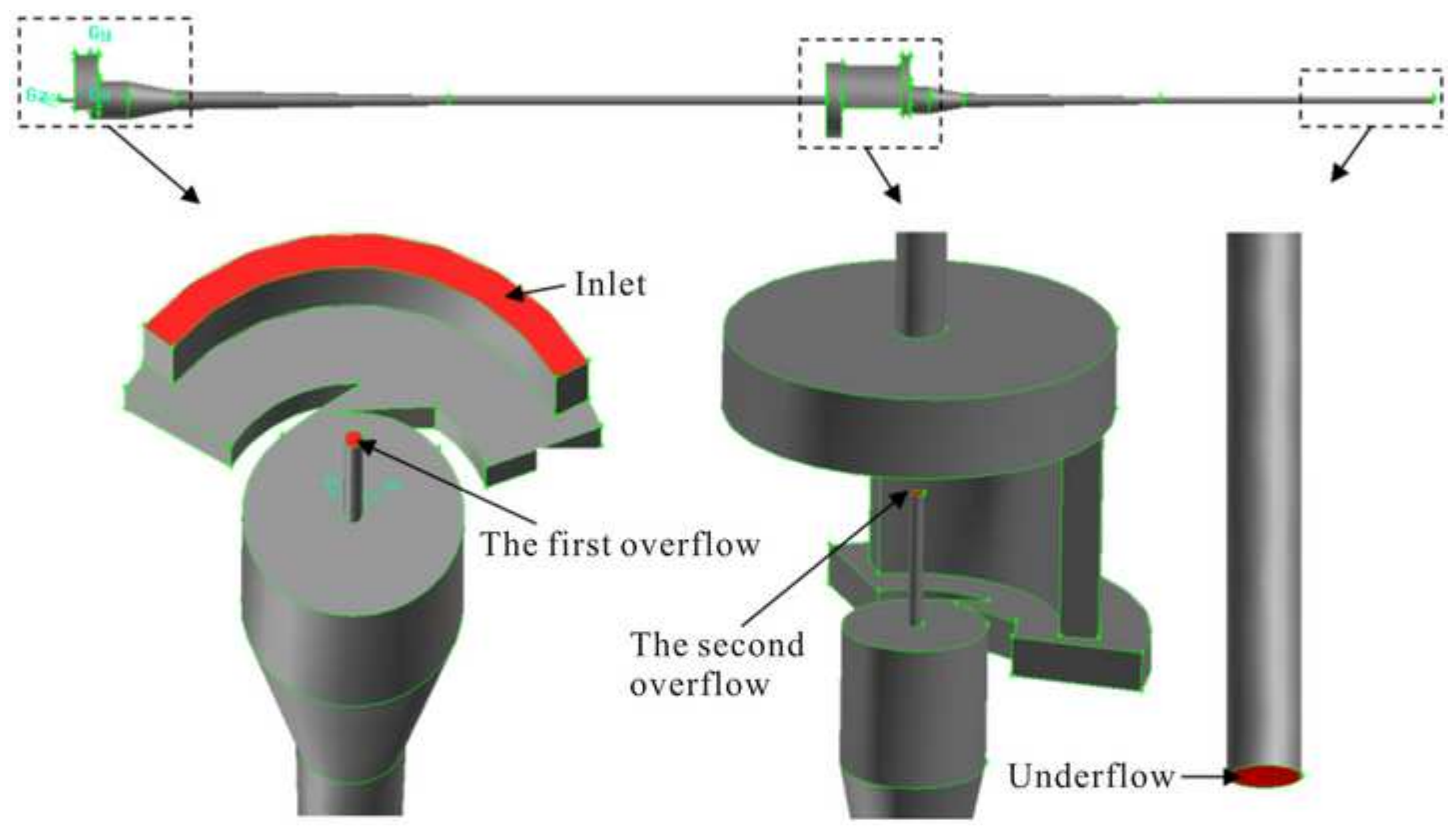

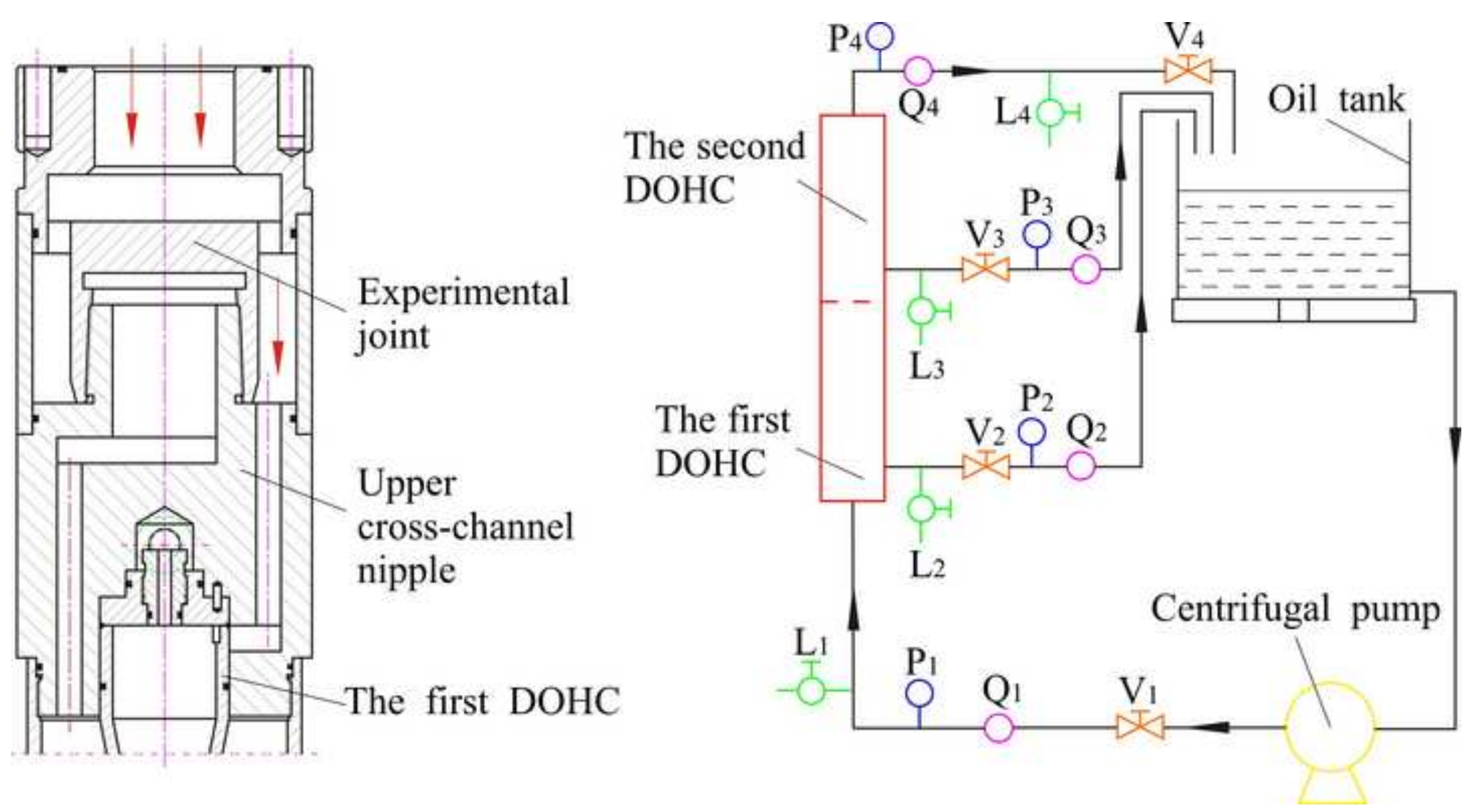


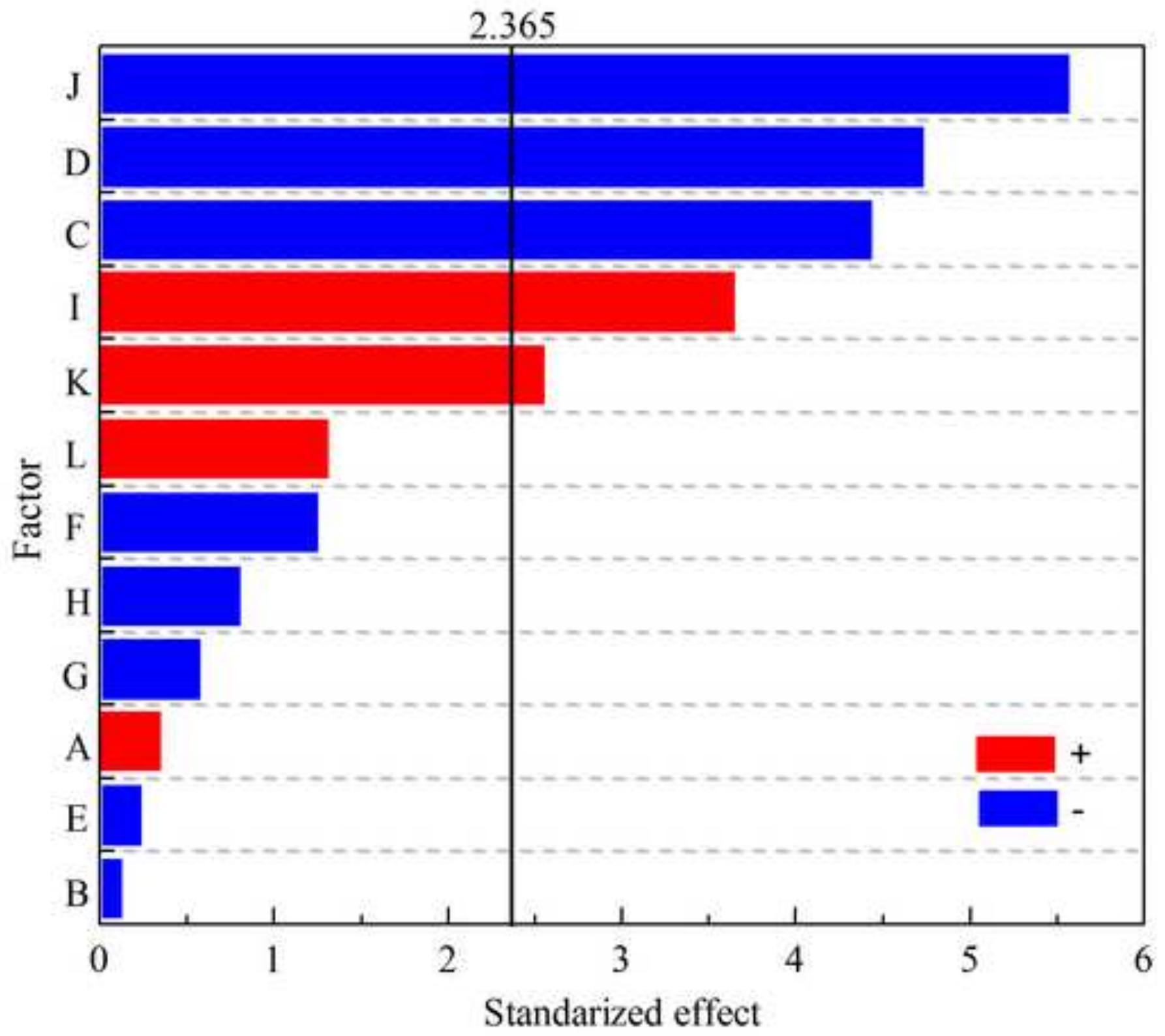




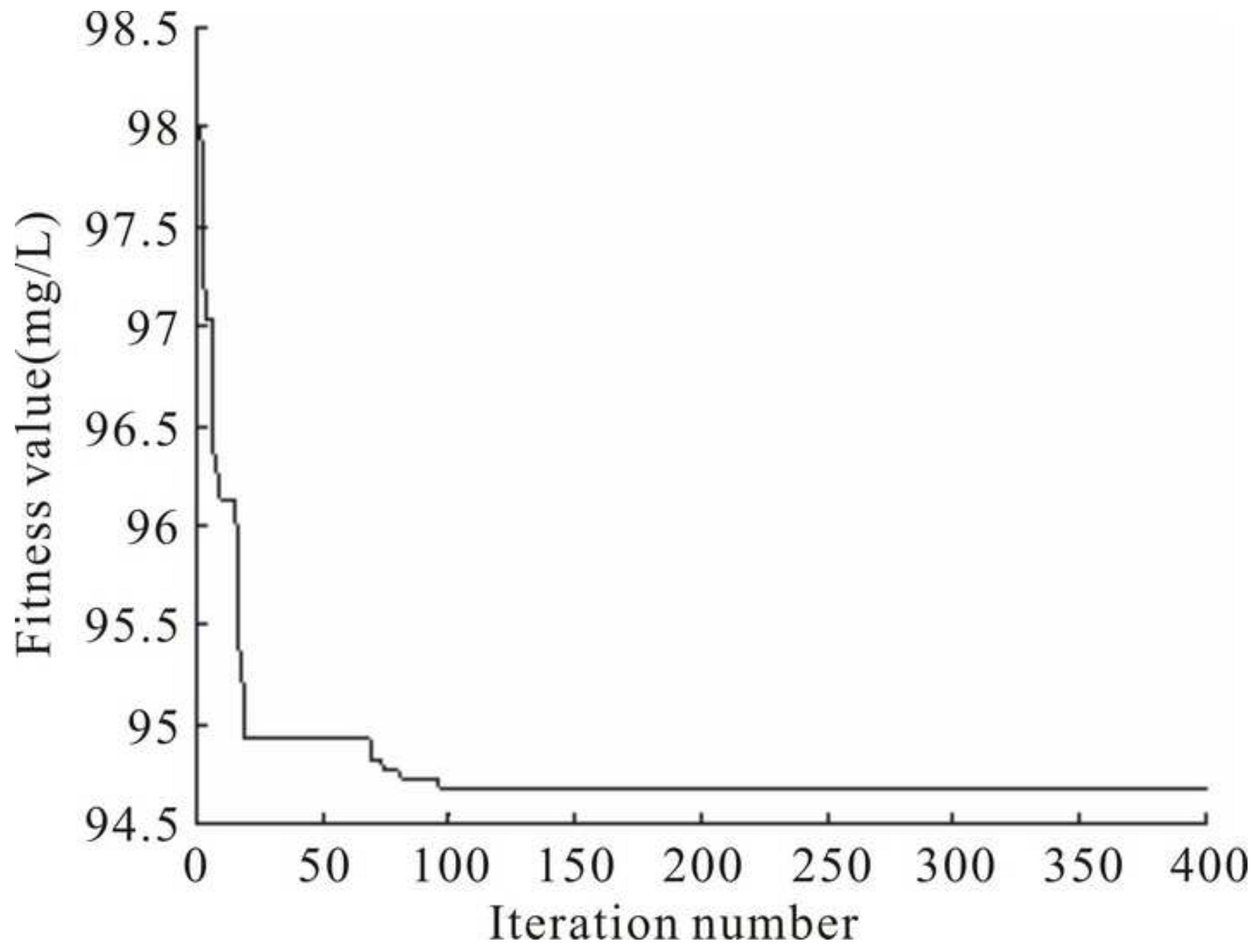




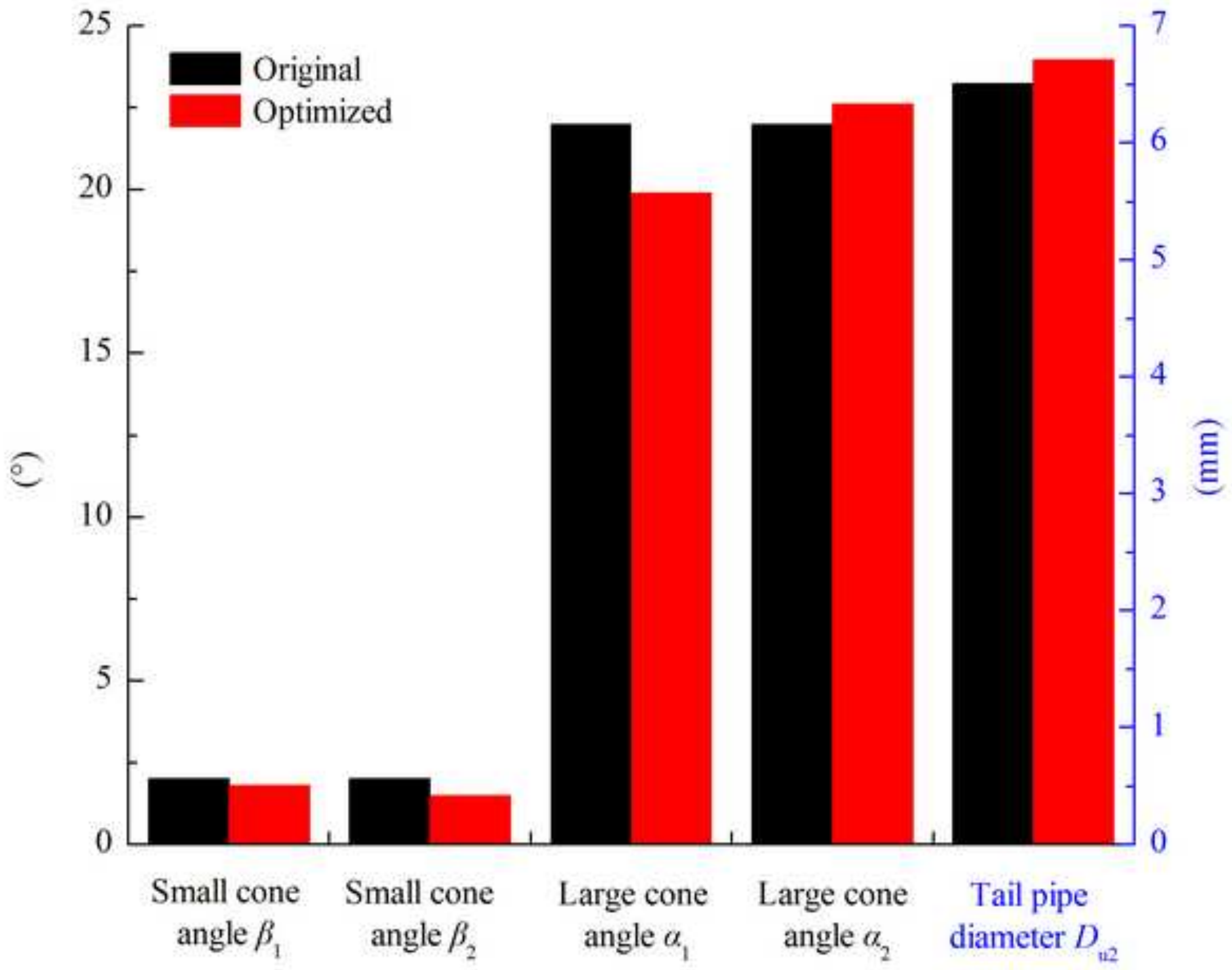



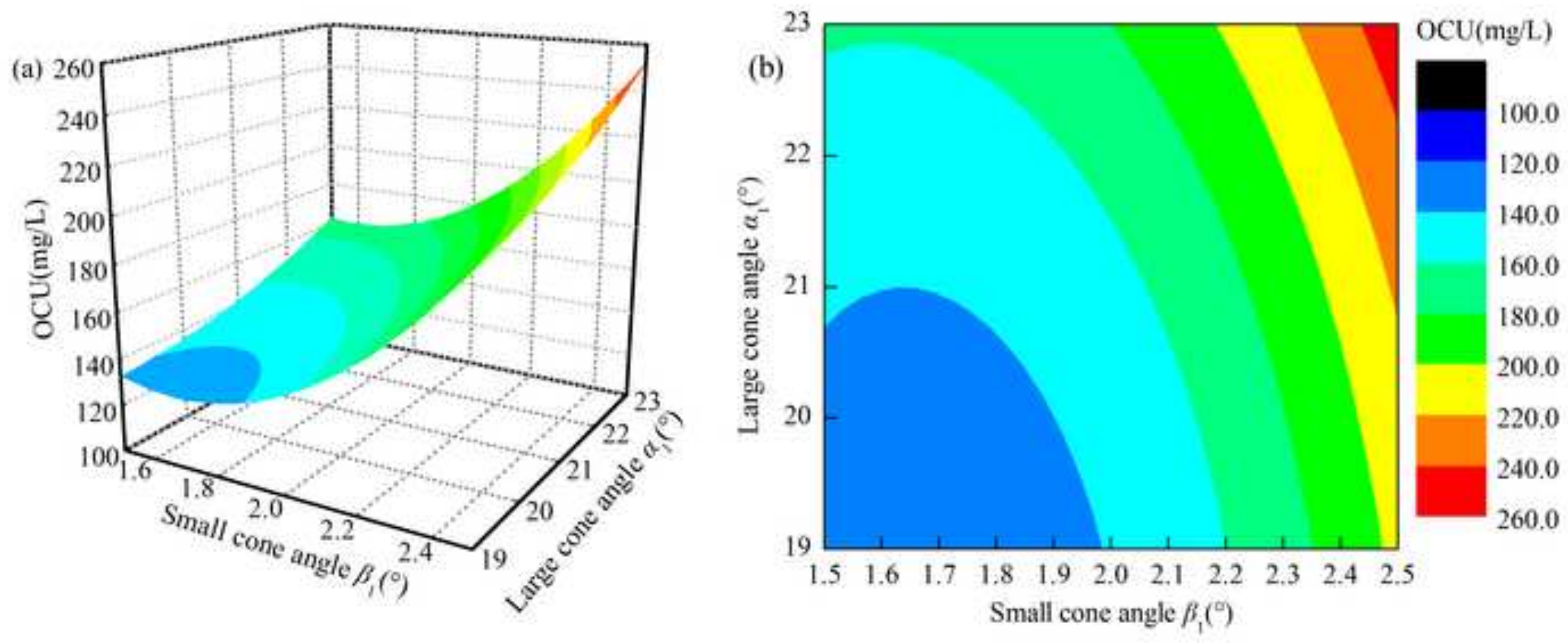

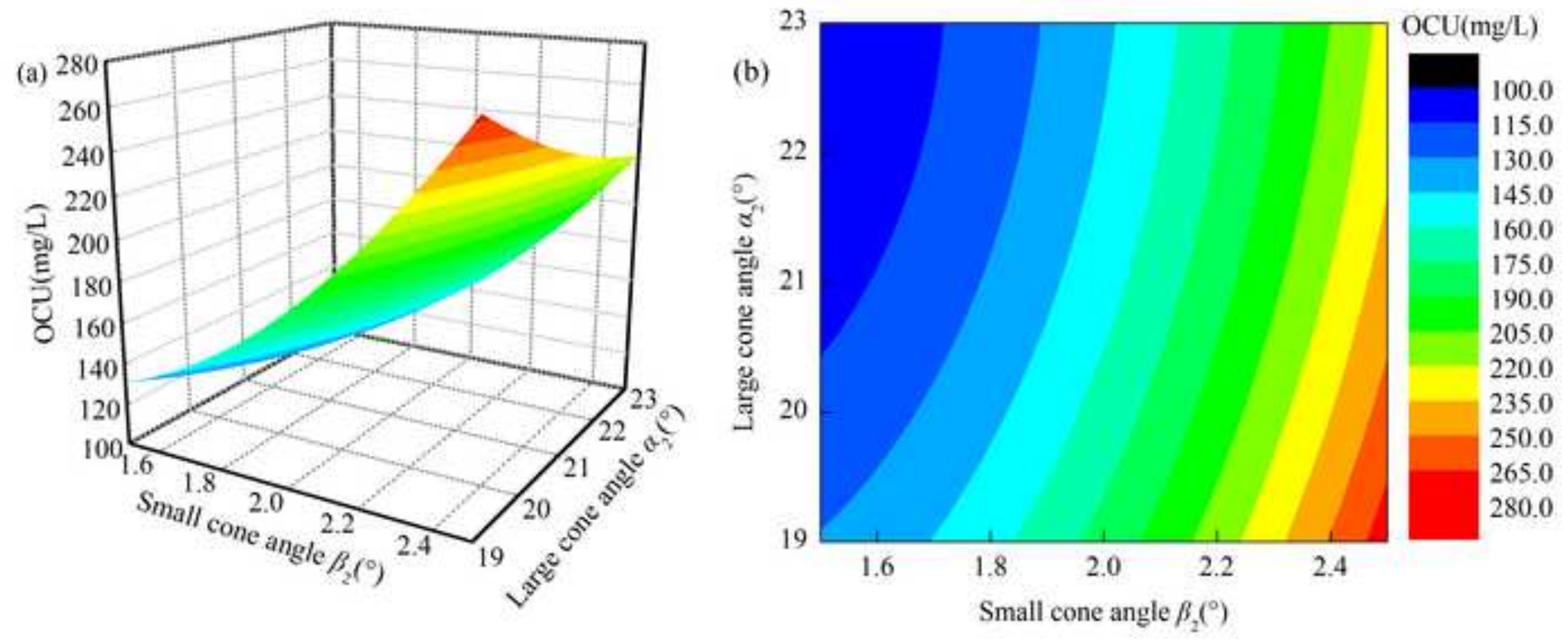

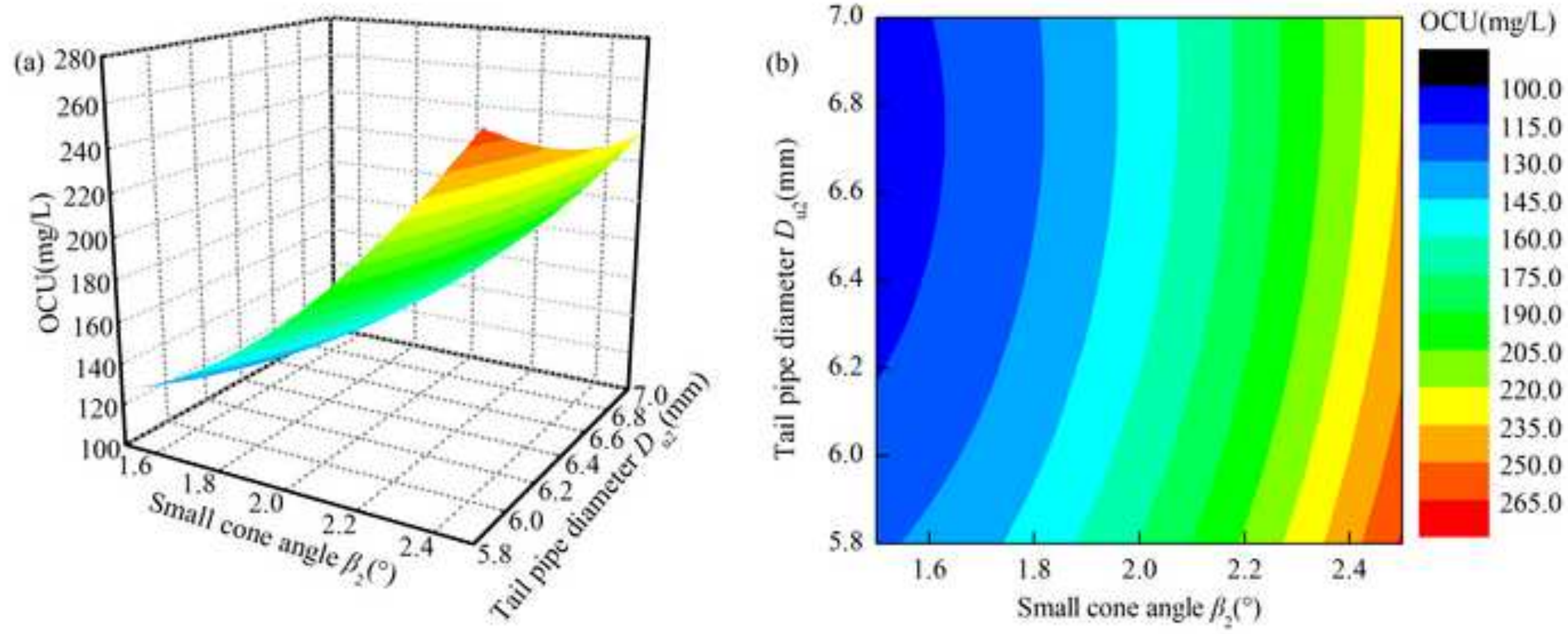

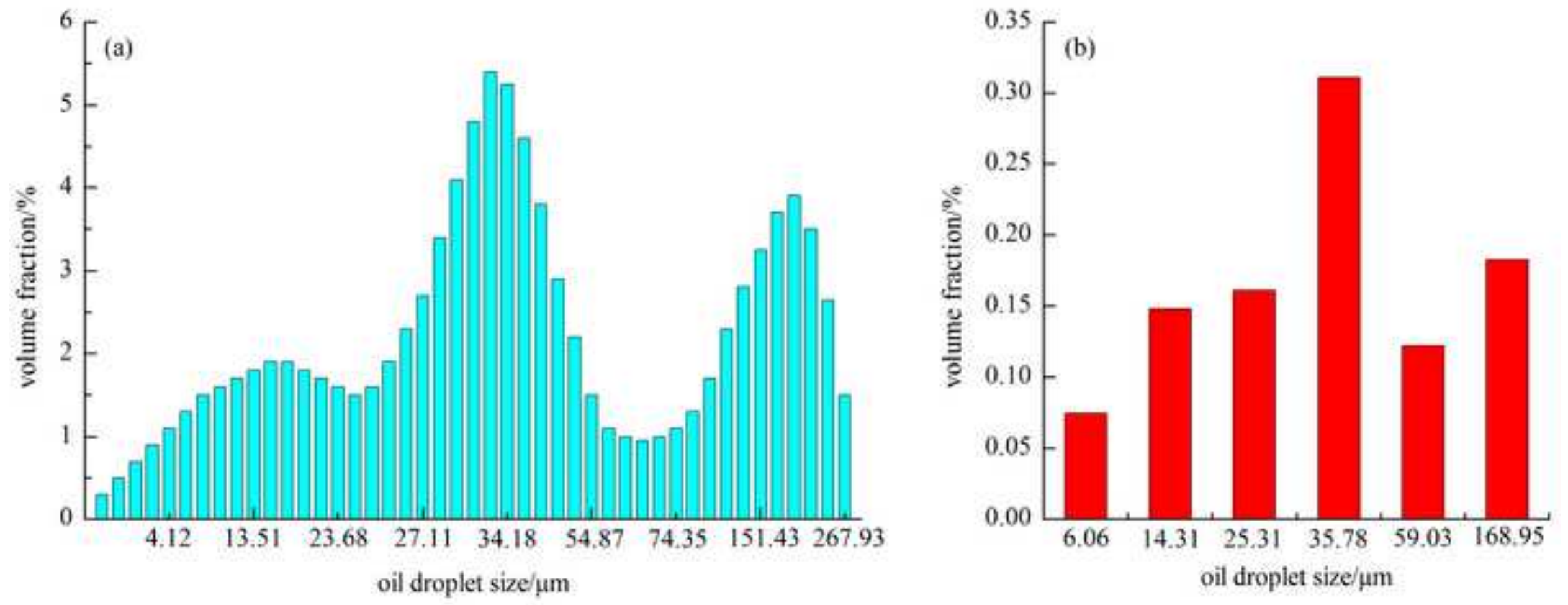


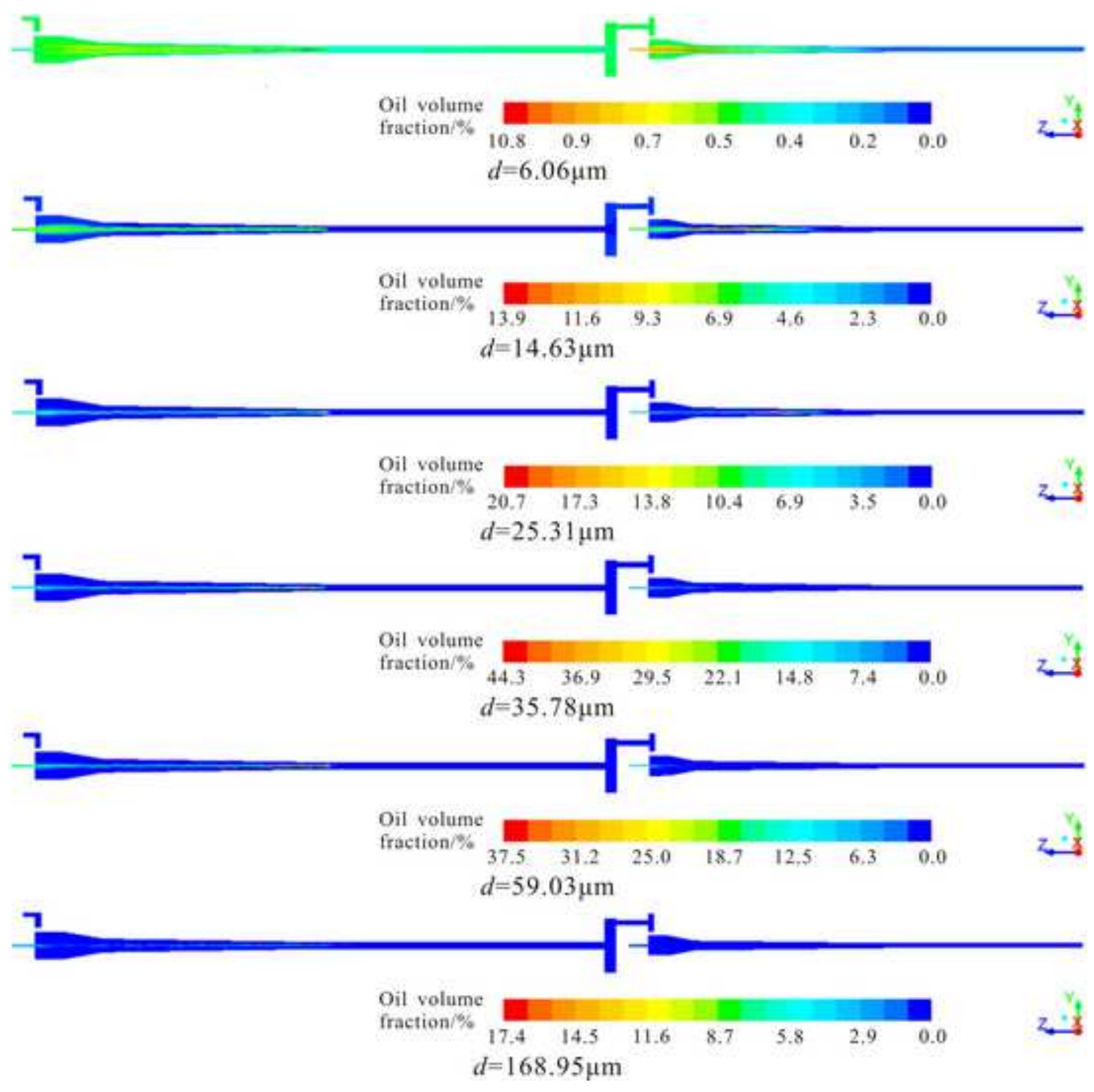




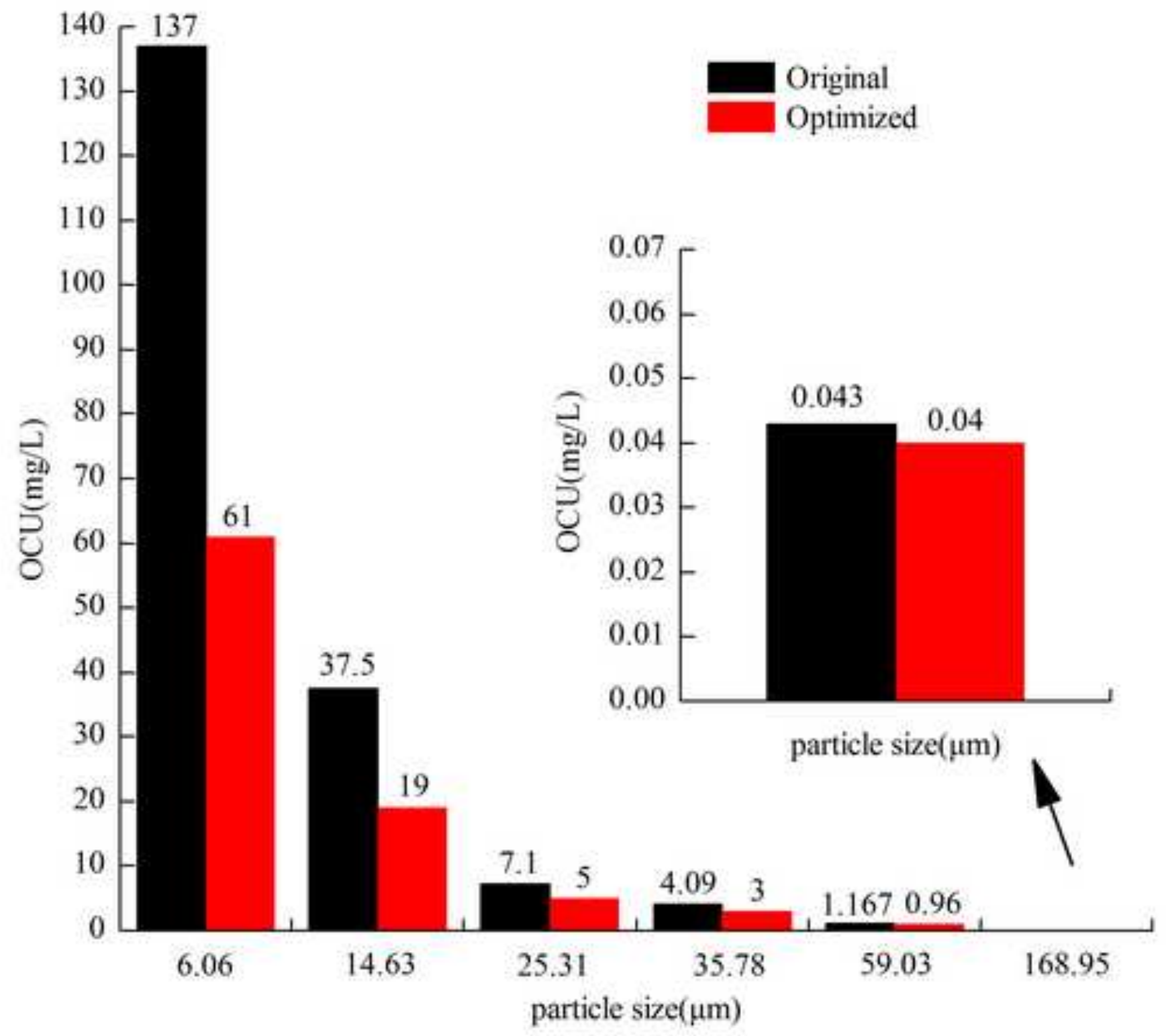

